\title{
A phase-field moving contact line model with soluble surfactants
}

2
Guangpu Zhu ${ }^{\mathrm{a}}$, Jisheng $\mathrm{Kou}^{\mathrm{b}, \text { d }}$, Jun Yao, and Aifen $\mathrm{Li}^{\mathrm{a}}$, *, Shuyu Sun ${ }^{\mathrm{c}, \text { * }}$

${ }^{a}$ School of Petroleum Engineering, China University of Petroleum (East China), Qingdao 266580, China

b School of Civil Engineering, Shaoxing University, Shaoxing 312000, Zhejiang, China.

${ }^{\mathrm{c}}$ Computational Transport Phenomena Laboratory, Division of Physical Science and Engineering, King Abdullah University of Science and Technology, Thuwal 23955-6900, Kingdom of Saudi Arabia

${ }^{\mathrm{d}}$ School of Mathematics and Statistics, Hubei Engineering University, Xiaogan 432000, Hubei, China.

* corresponding authors

Author information: b16020069@s.upc.edu.cn (Guangpu Zhu), shuyu.sun@kaust.edu.sa (Shuyu Sun)

keywords: phase-field modelling; moving contact line; surfactant; Navier-Stokes equation; energy stability

\section{Introduction}

Surfactants are usually amphiphilic compounds that can selectively absorb onto the interface and form a buffer zone to reduce the surface energy $[1,2]$. Surfactants play a crucial role in daily life and many industrial applications, such as water purification, detergents, oil-water emulsion in food processing and crude oil recovery [2,3]. Most recently, surfactants have been widely used in microsystems with the presence of interfaces, where the capillary effect dominates the inertia of fluids $[3,4]$. The presence of surfactants at the interface will significantly alter the dynamical behavior in microfluidic devices [2]. The computational modelling of interfacial dynamics with soluble surfactants remains a challenging task. Much effort has been devoted to simulating the multiphase system with surfactants, and several numerical methods have been developed, such as the level set method [5-7], the volume of fluid method [8, 9], the front tracking method[10], the immersed boundary method[3], the lattice Boltzmann method [2, 4, 11-13] and the phase-field 
method considered in this work [1,14-18]. The phase-field method is an effective modelling and simulation tool in the investigation of interfacial phenomena, and it has been extensively used with many successes [19-24]. In this method, a phase-field variable is introduced to distinguish two phases, and the interface is treated as a thin and continuous layer to remove singularities [25-29].

The phase-field method was used for the first time to study the interfacial flows with surfactants in [30]. Since then, numerous phase-field surfactant models have been proposed [1, 11, 31-33] and reviews of these models can refer to [34-36]. Further, a series of hydrodynamics coupled phase-field surfactant models were also developed [1, 2, 16, 18, 37, 38]. In the current work, a typical representative of nonlinearly coupled multivariate phase-field surfactant models in [1] is adopted. This model introduces a phase-field variable and a concentration field variable to represent the local densities of fluids and surfactant concentration, respectively. The logarithmic Floy-Huggins potential controls the entropy of mixing surfactants with bulk phases, and restricts the surfactant concentration between 0 and 1 [11]. A nonlinear coupling surface energy potential is used to account for the high concentration of surfactants along the fluid interface [35]. The enthalpic term in this model globally penalizes free surfactants in the respective phases and stabilizes the diffuse interface model $[1,11]$. This phase-field surfactant model can describe realistic adsorption isotherms, e.g., Langmuir isotherm, in thermodynamic equilibrium [1]. Recently, we coupled this model with the Navier-Stokes equation to simulate the interfacial dynamics with surfactants [39]. Several linear, decoupled and energy stable schemes were also constructed to solve this complex system effectively. Numerical results in both two and three dimensions demonstrate accuracy, efficiency and energy stability of the proposed schemes. We will try to extend this coupled model and schemes to the moving contact line (MCL) problem.

The MCL problem occurs when the fluid-fluid interface touches a solid surface [40-42]. It appears in the process of wetting, spray coating, and many biological applications [3]. Surfactants can significantly affect wetting properties by altering contact angles. For instance, a grease droplet can be easily removed from clothes if we add detergents (surfactants) into a washing machine [3]. A droplet sticking to clothes becomes less "sticky" under the effect of surfactants, and the water currents can wash away the droplet readily. In petroleum industry, surfactants and rock wettability determine the efficiency of water flooding and the distribution of residual oil in porous media [43]. Water, oil droplets, clothes (rock) and detergents (surfactants) make up a typical multiphase system involving the MCL and surfactants. The question then arises as to how surfactants affect the dynamics of MCL. In [3], the authors presented an immersed boundary method to simulate the MCL problem, and studied the effect of insoluble surfactants on the contact angles. In [6], a level set method for two-phase flows with the MCL and insoluble surfactants was proposed. Zhang et al. derived a continuous model for the dynamics of two immiscible fluids with the MCL and insoluble surfactants based on thermodynamic principles [44]. However, to the best of our knowledge, there is no diffuse interface model which takes both the MCL and surfactants into account.

To develop a phase-field MCL model with surfactants, we need to resolve the MCL conundrum for immiscible flows over the solid surface [41]. It is well known that under the usual hydrodynamic assumptions, namely the incompressible Navier-Stokes equation and no-slip boundary condition, non-physical singularities will occur in the vicinity of the MCL, and the velocity field is multivalued at the MCL [40-42]. Numerous models have been proposed over the years to describe the hydrodynamic behavior near the MCL, including the kinetic adsorption/desorption model, the slip models and the diffuse interface models $[41,45]$. Using careful molecular dynamics studies of the 
slip behavior near the MCL and principles of thermal dynamics, Qian et al proposed a generalized Navier boundary condition (GNBC) [46-49]. The GNBC states that the slip velocity at the MCL is proportional to the sum of the viscous stress and the unbalanced Young stress; the latter arises from the deviation of the fluid-fluid interface from its equilibrium configuration [41, 50]. They further demonstrated that the GNBC can quantitatively reproduce MCL slip velocity profiles obtained from MD simulations $[41,48]$. They also presented a variational derivation of the GNBC through the principle of the minimum energy dissipation, which indicated that the GNBC is consistent with the general principle of irreversible thermodynamics processes [48]. The GNBC shows great advantages and prospects in simulating the MCL dynamics, and several attempts have been made to develop energy stable schemes for the GNBC-based phase-field models [40, 42, 51, 52]. In this work, the GNBC is adopted to handle the slip boundary condition at the fluid-solid interface and the dynamic contact line condition.

As a first attempt, we present a phase-field model in this work for two-phase flows with the MCL and soluble surfactants. The nonlinearly coupled model consists of two Cahn-Hilliard-type equations, the Navier-Stokes equation and the GNBC. To solve such a complex governing system, we need to construct some energy stable numerical schemes. Recently, several efficient approaches have been proposed for the phase-field model, e.g., the convex splitting approach [40, 42], the stabilization approach [21,51], the invariant energy quadratization (IEQ) approach [39, 53] and the scalar auxiliary variable (SAV) approach $[28,54]$. The convex splitting approach explicitly treats the concave terms of energy functions and implicitly treats the convex terms. Although this approach has been successfully applied to construct unconditionally energy stable schemes for the phase-field model, it usually produces nonlinear schemes. For instance, this approach for the logarithmic FloryHuggins potential leads to the scheme involving the implicit logarithmic function. Thus, implementations of such schemes are often complicated and computational costs may be expensive accordingly. The stabilization method simply treats all nonlinear terms explicitly and introduces linear stabilization terms to remove the time step constraint. Schemes constructed by the stabilization approach are usually efficient, energy stable and easy-to-implement [22]. Recently, this approach shows great advantages in dealing with the nonlinear term from the MCL [51, 52]. However, this approach requires that the second derivative of the double-well potential to be bounded, which is not satisfied by the double well potential. The logarithmic potential is another challenge for the stabilization approach due to more complicate nonlinearity. The IEQ approach introduces some auxiliary variables to transform the free energy functional into an equivalent form [53], which allows nonlinear potentials to be treated efficiently and semi-explicitly. But this approach requires that nonlinear potentials are bounded from below, and this may not hold for some physical interesting models [54]. The main reason for this shortcoming is that auxiliary variables depend on space variables. The SAV approach almost shares the same idea with the IEQ approach but overcomes its shortcoming by using scalar auxiliary variables. This approach only requires that nonlinear parts of the free energy functional are bounded from below, which is necessary for the free energy to be physically sound [54]. A series of recent studies found that the SAV approach may be more accurate than the IEQ approach [28]. Most recently, the SAV approach has been applied to solve a large class of gradient flows due to its simplicity and efficiency [55]. In this work, we use the SAV approach to deal with the double well potential and the logarithmic potential. A stabilization term is artificially added to balance the explicit nonlinear term associated with the surface energy at the fluid-solid interface. A nonlinearly coupled scheme with unconditional energy stability is 
constructed to solve the transformed system. The proposed scheme is accurate and efficient. We further carry out a rigorous proof of energy stability.

The rest of this paper is organized as follows. In Section 2, we describe a phase-field MCL model with soluble surfactants. In Section 3, we transform this model into a new and an equivalent governing system by using the SAV approach. Then a nonlinearly coupled energy stable scheme for the transformed system is constructed, and the energy estimate is carried out for the proposed scheme. In Section 4, an efficient finite difference method on staggered grids is used for the spatial discretization. Several two and three dimensional numerical examples are investigated in Section 5 and the paper is finally concluded in Section 6.

\section{Governing equations}

In this section, we will describe a non-dimensional phase-field MCL model for a two-phase system with soluble surfactants (see the Appendix A for the original model and the definition of dimensionless numbers). The phase-field model introduces a phase-field variable and a concentration field variable to simulate the interfacial dynamics with surfactants. Governing equations for $\psi$ and $\phi$ take the form of Cahn-Hilliard-type equations [1, 2]

$$
\begin{gathered}
\psi_{t}+\nabla \cdot(\mathbf{u} \psi)=\frac{1}{\mathrm{Pe}_{\psi}} \nabla \cdot M_{\psi} \nabla w_{\psi}, \\
\phi_{t}+\nabla \cdot(\mathbf{u} \phi)=\frac{1}{\mathrm{Pe}_{\phi}} \Delta w_{\phi},
\end{gathered}
$$

where $\mathbf{u}$ is the velocity field, $M_{\psi}$ is the degenerate mobility, $\mathrm{Pe}_{\psi}$ and $\mathrm{Pe}_{\phi}$ are Péclet numbers. $\psi$ represents the surfactant concentration, and $\phi$ labels the local densities of fluids

$$
\phi(x, t)= \begin{cases}-1 & \text { fluid I, } \\ 1 & \text { fluid II. }\end{cases}
$$

The chemical potentials $w_{\psi}$ and $w_{\phi}$ are derived from the free energy functional $E_{f}[1]$

$$
E_{f}(\phi, \psi)=\int\left(F_{1}(\phi)+F_{2}(\psi)+F_{3}(\phi, \psi)+F_{4}(\phi, \psi)\right) d \Omega,
$$

where $F_{1}$ is the classical constant mobility Cahn-Hilliard potential

$$
F_{1}(\phi)=\frac{\mathrm{Cn}^{2}}{2}|\nabla \phi|^{2}+F(\phi)
$$

where $F(\phi)$ is the double well potential $\left(\phi^{2}-1\right)^{2} / 4$ and $\mathrm{Cn}$ is the Cahn number associated with the interfacial thickness. The gradient term in $F_{1}(\phi)$ promotes the mixing of fluids, and the polynomial part, double well potential, contributes to the separation of two phases. The competition between the two terms creates a diffuse interface in equilibrium [22].

$F_{2}$ is the Flory-Huggins potential [53] governing the entropy of mixing surfactants with the bulk phases

$$
\left\{\begin{array}{l}
F_{2}(\psi)=\operatorname{Pi} G(\psi), \\
G(\psi)=\psi \ln \psi+(1-\psi) \ln (1-\psi),
\end{array}\right.
$$

where Pi is the temperature-dependent constant taking the role of a diffusion coefficient for $\psi$. The first term $\psi \ln \psi$ in $G(\psi)$ models the ideal mixing of surfactants in the bulk phases and guarantees the value of $\psi$ to be positive, and the second term restricts $\psi<1$. Note that the degenerate mobility $M_{\psi}=$ $\psi(1-\psi)$, which vanishes at the extreme points $\psi=0$ and $\psi=1$, is introduced in equation (2.1) to combine with the logarithmic potential [1]. 
The special molecular composition of surfactants enables them to selectively absorb on the interface, and form a buffer zone to reduce the system energy. The surface energy potential $F_{3}$ accounts for the high concentration of surfactants near the interface

$$
F_{3}(\phi, \psi)=-\frac{\psi\left(\phi^{2}-1\right)^{2}}{4}
$$

The nonlinear coupling term $F_{4}$ is introduced to penalize free surfactants in the bulk phases.

$$
F_{4}(\phi, \psi)=\frac{\psi \phi^{2}}{2 \mathrm{Ex}}
$$

where the parameter Ex controls the bulk solubility. $F_{4}$ also plays a significant role in stabilizing the phase-field model. Obviously, $F_{4}$ is inactive on the interface where $\phi \approx 0$. To some extent, $F_{3}$ and $F_{4}$ are complementary: $F_{3}$ locally attracts surfactants to the interface while $F_{4}$ globally counteracts the occurrence of free surfactants [1].

Using the variational approach, we can obtain the chemical potentials $w_{\psi}$ and $w_{\phi}$,

$$
\begin{gathered}
w_{\psi}=\operatorname{Pi} \ln \left(\frac{\psi}{1-\psi}\right)+\frac{1}{2 \mathrm{Ex}} \phi^{2}-\frac{\left(\phi^{2}-1\right)^{2}}{4}, \\
w_{\phi}=-\phi+\phi^{3}-\mathrm{Cn}^{2} \Delta \phi+\frac{1}{\mathrm{Ex}} \psi \phi-\psi \phi\left(\phi^{2}-1\right) .
\end{gathered}
$$

The momentum equation for the hydrodynamics takes the usual form of the Navier-Stokes equation

$$
\begin{gathered}
\rho\left(\mathbf{u}_{t}+\mathbf{u} \cdot \nabla \mathbf{u}\right)+\mathbf{J} \cdot \nabla \mathbf{u}-\frac{1}{\operatorname{Re}} \nabla \cdot \eta D(\mathbf{u})+\nabla p+\frac{1}{\mathrm{We}}\left(\phi \nabla w_{\phi}+\psi \nabla w_{\psi}\right)=0, \\
\nabla \cdot \mathbf{u}=0
\end{gathered}
$$

where

$$
\mathbf{J}=\frac{\lambda_{\rho}-1}{2 \mathrm{Pe}_{\phi}} \nabla w_{\phi}, \quad \rho=\frac{1-\lambda_{\rho}}{2} \phi+\frac{1+\lambda_{\rho}}{2}, \quad \eta=\frac{1-\lambda_{\eta}}{2} \phi+\frac{1+\lambda_{\eta}}{2}, \quad \mathrm{We}=\mathrm{ReCaCn}
$$

here $\rho$ and $\eta$ are densities and viscosities of fluids, respectively. $\lambda_{\rho}$ and $\lambda_{\eta}$ are density and viscosity ratios, respectively. $p$ is the pressure, $\mathrm{Ca}$ is the capillary number, and $\mathrm{Re}$ is the Reynolds number.

The following conservation property $[22,51]$ can be easily derived from (2.2), (2.6) and (2.7):

$$
\rho_{t}+\nabla \cdot(\rho \mathbf{u})+\nabla \cdot \mathbf{J}=0
$$

If we consider extra body forces in the momentum equation, e.g., the gravitational force, the equation (2.5) can be modified into

$$
\rho\left(\mathbf{u}_{t}+\mathbf{u} \cdot \nabla \mathbf{u}\right)+\mathbf{J} \cdot \nabla \mathbf{u}-\frac{1}{\operatorname{Re}} \nabla \cdot \eta D(\mathbf{u})+\nabla p+\frac{1}{\operatorname{BoCn}}\left(\phi \nabla w_{\phi}+\psi \nabla w_{\psi}\right)-\rho \mathbf{g}=0,
$$

where Bo is the Bond number, and $\mathbf{g}$ is the unit vector denoting the direction of the body force.

When the fluid-fluid interface touches the solid wall, the MCL problem appears. The GNBC in this study is used to resolve the dynamics of MCL [40, 41]. The impermeability boundary condition requires normal components of diffusive fluxes and velocity at the fluid-solid interface to vanish,

$$
\partial_{n} w_{\phi}=\partial_{n} w_{\psi}=\mathbf{u} \cdot \mathbf{n}=0, \quad \text { on } \Gamma
$$

where $\Gamma$ denotes boundaries of the domain. The dynamic contact line condition for $\phi$ at the solid wall 
reads:

$$
\phi_{t}+\mathbf{u}_{\tau} \cdot \nabla_{\tau} \phi=-\frac{1}{\mathrm{Pe}_{s}} L(\phi), \quad \text { on } \Gamma,
$$

The slip boundary condition can be written as

$$
\frac{\mathrm{Ca} \eta}{L_{s}} \mathbf{u}_{s}=-\mathrm{Ca} \eta \partial_{n} \mathbf{u}_{\tau}+L \partial_{\tau} \phi, \quad \text { on } \Gamma,
$$

where $\mathbf{u}_{s}=\mathbf{u}_{\tau}-\mathbf{u}_{w}$ is the slip velocity of fluid on the solid wall, $\mathbf{u}_{w}$ is the wall velocity and $\mathbf{u}_{\tau}$ is the velocity along the tangential direction $\tau$ of solid wall. $\mathrm{Pe}_{s}$ is the positive phenomenological relaxation parameter and $L_{s}$ is the wall-fluid slip length. For the sake of simplicity, we will assume that two fluids have the same slip length in this study. The function $L(\phi)$ is defined as

$$
L(\phi)=\mathrm{Cn}_{n} \phi+M^{\prime}(\phi), \quad \text { on } \Gamma,
$$

and the surface energy at the fluid-solid interface $M(\phi)$ reads:

$$
M(\phi)=-\frac{\sqrt{2}}{3} \cos \theta_{s} \sin \left(\frac{\pi \phi}{2}\right), \quad \text { on } \Gamma,
$$

where $\theta_{s}$ is the static contact angle. The equation (2.9) means that the material derivative of $\phi$ at the solid surface is proportional to the deviation of $L(\phi)$ from its equilibrium value zero [41]. The equation (2.10) can be viewed as a force balance at the contact line [6]. The fraction force induced by the slip (left hand side) is equal to the sum of the viscous stress and the unbalance Young stress at the right hand side.

Equations (2.1) - (2.12) form a complete set of governing equations for the phase-field MCL model with soluble surfactants, and the total energy of the hydrodynamic system is the sum of the kinetic energy, the free energy $E_{f}$ and the surface energy at the fluid-solid interface

$$
\begin{gathered}
E_{t o t}(\mathbf{u}, \phi, \psi)=\frac{\mathrm{We}}{2}\left(\rho,|\mathbf{u}|^{2}\right)+\frac{\mathrm{Cn}^{2}}{2}\|\nabla \phi\|^{2}+(F(\phi), 1)+\operatorname{Pi}(G(\psi), 1) \\
+\frac{1}{2 \mathrm{Ex}}\left(\psi, \phi^{2}\right)-\frac{1}{4}\left(\psi,\left(\phi^{2}-1\right)^{2}\right)+\operatorname{Cn}(M(\phi), 1)_{\Gamma} .
\end{gathered}
$$

It can be observed that each term in the total energy $E_{\text {tot }}$ is bounded from below, since the phasefield variable $\phi$ varies from -1 to 1 , and the concentration variable $\psi$ is restricted in the domain $(0$, 1). Thus, the total energy $E_{\text {tot }}$ has a lower bound. We can also easily derive the following PDE energy dissipation law for the governing system (2.1) - (2.12).

$$
\begin{gathered}
\frac{d}{d t} E_{\text {tot }}(\mathbf{u}, \phi, \psi)=-\frac{1}{\mathrm{Pe}_{\phi}}\left\|\nabla w_{\phi}\right\|^{2}-\frac{1}{\mathrm{Pe}_{\psi}}\left\|\sqrt{M_{\psi}} \nabla w_{\psi}\right\|^{2}-\frac{\mathrm{CaCn}}{2}\|\sqrt{\eta} D(\mathbf{u})\|^{2} \\
-\frac{\mathrm{Cn}}{\mathrm{Pe}_{s}}\|L(\phi)\|_{\Gamma}^{2}-\frac{\mathrm{CaCn}}{L_{s}}\left\|\sqrt{\eta} \mathbf{u}_{s}\right\|_{\Gamma}^{2} \leq 0 .
\end{gathered}
$$

As in [1], we now analyze the equilibrium property of surfactant adsorption. In equilibrium, the entire system has the same chemical potential $\left(w_{\psi}=C, C\right.$ being a constant). Thus, we have $w_{\psi_{B}}=w_{\psi}$.

$$
\begin{aligned}
& w_{\psi_{B}}=\operatorname{Piln}\left(\frac{\psi_{B}}{1-\psi_{B}}\right)+\frac{1}{2 \mathrm{Ex}} \phi_{B}^{2}-\frac{\left(\phi_{B}^{2}-1\right)^{2}}{4}, \\
& w_{\psi}=\operatorname{Pi} \ln \left(\frac{\psi}{1-\psi}\right)+\frac{1}{2 \mathrm{Ex}} \phi^{2}-\frac{\left(\phi^{2}-1\right)^{2}}{4},
\end{aligned}
$$


where $\psi_{B}$ is the surfactant bulk concentration and is much smaller than unity $\left(\psi_{b} \leq 1\right)$, and $\phi_{B}$ is the phase bulk value ( $\phi_{B}$ roughly equals \pm 1 ). Subtracting (2.13) from (2.14), and introducing the intermediate variable $\psi_{c}(x)$, we get the relation

$$
\operatorname{Pi} \ln \psi_{c}=-\frac{1}{2 \mathrm{Ex}}\left(\phi_{B}^{2}-\phi^{2}\right)-\frac{1}{4}\left[\left(\phi_{B}^{2}-\phi^{2}\right)\left(2-\phi_{B}^{2}-\phi^{2}\right)\right],
$$

then the steady-state profile for $\psi$ can be obtained

$$
\psi=\frac{\psi_{B}}{\psi_{B}+\psi_{c}\left(1-\psi_{B}\right)} .
$$

Using the assumption that $\phi=0$ on the interface of fluids, $\phi_{B}= \pm 1$ and $\psi_{B}$ is small, we can simplify (2.16) and (2.15) as

(1)

$$
\begin{aligned}
\psi_{0} & =\frac{\psi_{B}}{\psi_{B}+\psi_{c}}, \\
\operatorname{Pi} \ln \psi_{c} & =-\frac{1}{4}\left(1+\frac{2}{\mathrm{Ex}}\right),
\end{aligned}
$$

where $\psi_{0}$ is the equilibrium concentration of surfactants on the phase interface. Equation (2.17) is the typical Langmuir isotherm and $\psi_{c}$ is the Langmuir adsorption constant [1]. Given specific Pi (temperature-dependent constant) and Ex, $\psi_{c}$ can be obtained. The adsorption isotherm is important for the following simulations. Next we will try to construct an efficient time marching scheme with unconditional energy stability to solve such complex system.

\section{Numerical schemes}

\subsection{Transformed governing system and its energy law}

The main challenge in solving phase-field models is to develop efficient schemes with unconditional energy stability. Numerical schemes that do not respect energy dissipation laws may be "overloaded" with an excessive amount dissipation near singularities, which in turn lead to large numerical errors, particularly for long time integration [22, 56]. Hence, to accurately simulate the interfacial dynamics with surfactants, it is especially desirable to design a scheme that satisfies the discrete energy law. There are several difficulties $[22,57]$ in constructing such a scheme, including (1) the nonlinear couplings between $\phi$ and $\psi$; (2) the nonlinear terms associated with nonlinear potentials, such as the double well potential and Flory-Huggins potential; (3) the nonlinear couplings between $\phi, \psi$ and the velocity $\mathbf{u}$ through stress and convection terms; (4) the coupling between the velocity and pressure; (5) the nonlinear couplings between the velocity and the phase-field variable $\phi$ at the MCL. For the difficulties (1) and (2), a newly developed SAV approach is adopted to handle the nonlinear terms. The SAV approach introduces some scalar auxiliary variables to transform the free energy into an equivalent form, which provides fundamental support for the semi-explicit treatment [54]. The SAV approach has been successfully applied to a large class of gradient flows due to its simplicity and efficiency [55]. Most recently, this approach has been extended to the case of multiple nonlinearly coupled variables [36]. For the difficulty (3), we use some subtle implicitexplicit treatments to stress and convection terms. For the difficulty (4), a splitting method based on the pressure stabilization is implemented to decouple the computation of velocity from pressure. For the last difficulty, a stabilization term is added to balance the explicit nonlinear term associated with the surface energy at the fluid-solid interface.

As in $[53,58]$, we first regularize the logarithmic potential $G(\psi)$ from the domain $(0,1)$ to $(-\infty$, 
$+\infty)$. For any $\xi>0$, the regularized logarithmic potential can be written as

$$
\hat{G}(\psi)=\left\{\begin{array}{lr}
\psi \ln \psi+\frac{(1-\psi)^{2}}{2 \zeta}+(1-\psi) \ln \zeta-\frac{\zeta}{2}, & \text { if } \quad \psi \geq 1-\zeta, \\
\psi \ln \psi+(1-\psi) \ln (1-\psi), & \text { if } \quad \zeta \leq \psi \leq 1-\zeta, \\
(1-\psi) \ln (1-\psi)+\frac{\psi^{2}}{2 \zeta}+\psi \ln \zeta-\frac{\zeta}{2}, & \text { if } \quad \psi \leq \zeta .
\end{array}\right.
$$

3 When $\zeta \rightarrow 0, \hat{G}(\psi) \rightarrow G(\psi)$, and we consider the numerical solution to the model formulated with 4 the regularized function $\hat{G}(\psi)$. The^ is omitted in the notation for convenience.

Following the idea of SAV approach, we define two scalar auxiliary variables [53]

$$
U=\sqrt{E_{u}(\phi)}, \quad V=\sqrt{E_{v}(\psi)},
$$

where

$$
E_{u}(\phi)=\int F(\phi) d \Omega, \quad E_{v}(\psi)=\int(G(\psi)+B) d \Omega,
$$

here $B$ is a constant to ensure $G(\psi)+B>0$. We adopt $B=1$ in this study. Then the total energy can be rewritten as

$$
\begin{aligned}
& E_{\text {tot }}(\mathbf{u}, \phi, \psi, U, V)=\frac{\mathrm{We}}{2}\left(\rho,|\mathbf{u}|^{2}\right)+\frac{\mathrm{Cn}^{2}}{2}\|\nabla \phi\|^{2}+\frac{1}{2 \mathrm{Ex}}\left(\psi, \phi^{2}\right)-\frac{1}{4}\left(\psi,\left(\phi^{2}-1\right)^{2}\right) \\
& \quad+\operatorname{Cn}(M(\phi), 1)_{\Gamma}+U^{2}+\operatorname{Pi} V^{2}-\operatorname{Pi} B|\Omega| .
\end{aligned}
$$

Note that the total free energy $E_{t o t}$ is not changed due to the introduction of zero terms $\mathrm{Pi} B|\Omega|-\mathrm{Pi} B|\Omega|$. Using the variational approach, a new and an equivalent governing system can be obtained

$$
\begin{gathered}
\psi_{t}+\nabla \cdot(\mathbf{u} \psi)=\frac{1}{\mathrm{Pe}_{\psi}} \nabla \cdot M_{\psi} \nabla w_{\psi}, \\
w_{\psi}=\frac{\operatorname{Pi} V}{\sqrt{E_{v}(\psi)}} G^{\prime}(\psi)+\frac{\phi^{2}}{2 \mathrm{Ex}}-\frac{W^{2}}{4},
\end{gathered}
$$

$$
V_{t}=\frac{1}{2 \sqrt{E_{v}(\psi)}} \int G^{\prime}(\psi) \psi_{t} d \Omega
$$

$$
\phi_{t}+\nabla \cdot(\mathbf{u} \phi)=\frac{1}{\mathrm{Pe}_{\phi}} \Delta w_{\phi}
$$

(1)

$$
w_{\phi}=\frac{U}{\sqrt{E_{u}(\phi)}} F^{\prime}(\phi)-\mathrm{Cn}^{2} \Delta \phi+\frac{\psi \phi}{\mathrm{Ex}}-\psi \phi W,
$$

$$
\rho\left(\mathbf{u}_{t}+\mathbf{u} \cdot \nabla \mathbf{u}\right)+\mathbf{J} \cdot \nabla \mathbf{u}-\frac{1}{\operatorname{Re}} \nabla \cdot \eta D(\mathbf{u})+\nabla p+\frac{1}{\mathrm{We}}\left(\phi \nabla w_{\phi}+\psi \nabla w_{\psi}\right)=0,
$$

$$
\nabla \cdot \mathbf{u}=0
$$

with boundary conditions

$$
\partial_{n} w_{\phi}=0, \quad \partial_{n} w_{\psi}=0, \quad \mathbf{u} \cdot \mathbf{n}=0, \quad \text { on } \Gamma,
$$


3 where

5 and

$$
\mathbf{J}=\frac{\lambda_{\rho}-1}{2 \mathrm{Pe}_{\phi}} \nabla w_{\phi}, \quad \rho=\frac{1-\lambda_{\rho}}{2} \phi+\frac{1+\lambda_{\rho}}{2}, \quad \eta=\frac{1-\lambda_{\eta}}{2} \phi+\frac{1+\lambda_{\eta}}{2} .
$$

To facilitate the proof of energy stability, here we denote $\phi^{2}-1$ as $W$ in equation (3.4) and (3.7). Now we can derive the PDE energy law for the transformed governing system (3.3) - (3.15). Assuming $\mathbf{u}_{w}=0$, then the transformed governing system satisfies the following energy dissipation law (the detailed derivation can refer to the Appendix B)

$$
\begin{gathered}
\frac{d}{d t} E_{t o t}(\mathbf{u}, \phi, \psi, U, V)=-\frac{1}{\mathrm{Pe}_{\phi}}\left\|\nabla w_{\phi}\right\|^{2}-\frac{1}{\mathrm{Pe}_{\psi}}\left\|\sqrt{M_{\psi}} \nabla w_{\psi}\right\|^{2}-\frac{\mathrm{CaCn}}{2}\|\sqrt{\eta} D(\mathbf{u})\|^{2} \\
-\frac{\mathrm{Cn}}{\mathrm{Pe}_{s}}\|L(\phi)\|_{\Gamma}^{2}-\frac{\mathrm{CaCn}}{L_{s}}\left\|\sqrt{\eta} \mathbf{u}_{s}\right\|_{\Gamma}^{2} \leq 0,
\end{gathered}
$$

where

$$
\begin{aligned}
E_{\text {tot }}(\mathbf{u}, \phi, \psi, U, V)= & \frac{\mathrm{We}}{2}\left(\rho,|\mathbf{u}|^{2}\right)+\frac{\mathrm{Cn}^{2}}{2}\|\nabla \phi\|^{2}+\frac{1}{2 \mathrm{Ex}}\left(\psi, \phi^{2}\right)-\frac{1}{4}\left(\psi,\left(\phi^{2}-1\right)^{2}\right) \\
& +\mathrm{Cn}(M(\phi), 1)_{\Gamma}+U^{2}+\operatorname{Pi} V^{2}-\operatorname{Pi} B|\Omega| .
\end{aligned}
$$

It is obvious that the transformed phase-field MCL model with soluble surfactants satisfies the exactly same energy dissipation law with the original system for the time-continuous case. In the next section, we will focus on constructing an energy stable time-marching scheme for the transformed governing system.

\subsection{Numerical scheme with unconditional energy stability}

In this section, an energy stable scheme is presented to solve the governing system (3.3) (3.15). We first define the following variable

$$
L_{1}:=\max \left|M^{\prime \prime}(\phi)\right|=\frac{\sqrt{2} \pi^{2}}{12} .
$$

To deal with undershoot and overshoot values around the interface, a cut-off function is defined [22]

$$
\widehat{\phi}^{n+1}= \begin{cases}\phi^{n+1} & \left|\phi^{n+1}\right| \leq 1, \\ \operatorname{sign}\left(\phi^{n+1}\right) & \left|\phi^{n+1}\right|>1 .\end{cases}
$$

Given $\psi^{n}, \phi^{n}, V^{n}, U^{n}, \mathbf{u}^{n}$ and $p^{n}$, the scheme (3.19) - (3.31) calculates $\psi^{n+1}, \phi^{n+1}, V^{n+1}, U^{n+1}, \mathbf{u}^{n+1}$ and $p^{n+1}$ for $n \geq 0$ in two steps.

Step 1, we update $\psi^{n+1}, \phi^{n+1}, \mathbf{u}^{n+1}, V^{n+1}$, and $U^{n+1}$ by solving

$$
\frac{\psi^{n+1}-\psi^{n}}{\delta t}+\nabla \cdot\left(\mathbf{u}^{n+1} \psi^{n}\right)-\frac{1}{\mathrm{Pe}_{\psi}} \nabla \cdot M_{\psi}^{n} \nabla w_{\psi}^{n+1}=0,
$$




$$
\begin{gathered}
w_{\psi}^{n+1}=\frac{\operatorname{Pi} V^{n+1}}{\sqrt{E_{v}\left(\psi^{n}\right)}} G^{\prime}\left(\psi^{n}\right)+\frac{\left(\phi^{n}\right)^{2}}{2 \mathrm{Ex}}-\frac{\left(W^{n}\right)^{2}}{4}, \\
\frac{V^{n+1}-V^{n}}{\delta t}=\frac{1}{2 \sqrt{E_{v}\left(\psi^{n}\right)}} \int G^{\prime}\left(\psi^{n}\right) \frac{\psi^{n+1}-\psi^{n}}{\delta t} d \Omega, \\
\frac{\phi^{n+1}-\phi^{n}}{\delta t}+\nabla \cdot\left(\mathbf{u}^{n+1} \phi^{n}\right)-\frac{1}{\mathrm{Pe}_{\phi}} \Delta w_{\phi}^{n+1}=0, \\
w_{\phi}^{n+1}=\frac{U^{n+1}}{\sqrt{E_{u}\left(\phi^{n}\right)}} F^{\prime}\left(\phi^{n}\right)-\mathrm{Cn}^{2} \Delta \phi^{n+1}+\frac{\psi^{n+1} \phi^{n+1}}{\mathrm{Ex}}-\frac{1}{2} \psi^{n+1} W^{n}\left(\phi^{n+1}+\phi^{n}\right), \\
\frac{U^{n+1}-U^{n}}{\delta t}=\frac{1}{2 \sqrt{E_{u}\left(\phi^{n}\right)}} \int F^{\prime}\left(\phi^{n}\right) \frac{\phi^{n+1}-\phi^{n}}{\delta t} d \Omega,
\end{gathered}
$$

with boundary conditions

$$
\rho^{n} \frac{\mathbf{u}^{n+1}-\mathbf{u}^{n}}{\delta t}-\frac{1}{\operatorname{Re}} \nabla \cdot \eta^{n} D\left(\mathbf{u}^{n+1}\right)+\nabla\left(2 p^{n}-p^{n-1}\right)+\rho^{n}\left(\mathbf{u}^{n} \cdot \nabla\right) \mathbf{u}^{n+1}+\mathbf{J}^{n} \cdot \nabla \mathbf{u}^{n+1}+
$$

$$
\frac{1}{\mathrm{We}}\left(\phi^{n} \nabla w_{\phi}^{n+1}+\psi^{n} \nabla w_{\psi}^{n+1}\right)+\frac{1}{2} \frac{\rho^{n+1}-\rho^{n}}{\delta t} \mathbf{u}^{n+1}+\frac{1}{2} \nabla \cdot\left(\rho^{n} \mathbf{u}^{n}\right) \mathbf{u}^{n+1}+\frac{1}{2} \nabla \cdot \mathbf{J}^{n} \mathbf{u}^{n+1}=0,
$$

where

$$
\begin{gathered}
\partial_{n} w_{\psi}^{n+1}=\partial_{n} w_{\phi}^{n+1}=\mathbf{u}^{n+1} \cdot \mathbf{n}=0, \quad \text { on } \Gamma, \\
\frac{\phi^{n+1}-\phi^{n}}{\delta t}+\mathbf{u}_{\tau}^{n+1} \cdot \nabla_{\tau} \phi^{n}=-\frac{1}{\mathrm{Pe}_{s}} L^{n+1}, \quad \text { on } \Gamma,
\end{gathered}
$$$$
\frac{\mathrm{Ca} \eta^{n}}{L_{s}} \mathbf{u}_{s}^{n+1}+\mathrm{Ca} \eta^{n} \partial_{n} \mathbf{u}_{\tau}^{n+1}-L^{n+1} \nabla_{\tau} \phi^{n}=0, \quad \text { on } \Gamma,
$$

$$
\mathbf{J}^{n}=\frac{\lambda_{\rho}-1}{2 \mathrm{Pe}_{\phi}} \nabla w_{\phi}^{n}, \quad \rho^{n+1}=\frac{1-\lambda_{\rho}}{2} \widehat{\phi}^{n+1}+\frac{1+\lambda_{\rho}}{2}, \eta^{n+1}=\frac{1-\lambda_{\eta}}{2} \widehat{\phi}^{n+1}+\frac{1+\lambda_{\eta}}{2} .
$$

and

$$
L^{n+1}=\mathrm{Cn} \partial_{n} \phi^{n+1}+M^{\prime}\left(\phi^{n}\right)+S\left(\phi^{n+1}-\phi^{n}\right), \quad \text { on } \Gamma,
$$

where $S$ is a positive constant with the magnitude determined later.

Step 2, to avoid solving an elliptic equation with $1 / \rho$ as the variable coefficient, we use a pressure stabilization scheme to solve the pressure $[22,59]$, which leads to a pressure Poisson equation

$$
\left\{\begin{array}{l}
-\Delta\left(p^{n+1}-p^{n}\right)=-\frac{\chi}{\delta t} \nabla \cdot \mathbf{u}^{n+1}, \\
\nabla p^{n+1} \cdot \mathbf{n}=0, \quad \text { on } \Gamma,
\end{array}\right.
$$

where $\chi=\frac{1}{2} \min \left(1, \lambda_{\rho}\right)$.

Remark 3.1. (1) We can see that the introduction of $U$ and $V$ allows the double well potential and logarithmic potential to be treated semi-implicitly. Compared with the traditional approaches, e.g., the convex splitting approach, the SAV approach only requires the nonlinear parts of free energy are bounded from below, which is necessary for the free energy to be physically sound. Also, this approach is not restricted to the specific forms of nonlinear parts of free energy, and it works well for the double well potential and logarithmic potential in this study. (2) The last three terms in (3.25) is the first-order approximation of the term 


$$
\frac{1}{2}\left(\rho_{t}+\nabla \cdot(\rho \mathbf{u})+\nabla \cdot \mathbf{J}\right) \mathbf{u}
$$

at $t^{n+1}$. These terms vanish in equation (3.9) due to $\rho_{t}+\nabla \cdot(\rho \mathbf{u})+\nabla \cdot \mathbf{J}$ equals 0 [51].

Next we will carry out the energy estimates for the above scheme.

Theorem 3.2. Assuming $\mathbf{u}_{w}=0$ and $S \geq L_{1} / 2$, then the scheme (3.19) - (3.31) is unconditionally energy stable, and satisfies the following discrete energy law:

$$
\begin{aligned}
\frac{E_{\text {tot }}^{n+1}-E_{\text {tot }}^{n}}{\delta t} & \leq-\frac{1}{\mathrm{Pe}_{\psi}}\left\|\sqrt{M_{\psi}^{n}} \nabla w_{\psi}^{n+1}\right\|^{2}-\frac{1}{\mathrm{Pe}_{\phi}}\left\|\nabla w_{\phi}^{n+1}\right\|^{2}-\frac{\mathrm{CaCn}}{2}\left\|\sqrt{\eta^{n}} D\left(\mathbf{u}^{n+1}\right)\right\|^{2} \\
& -\frac{\mathrm{CaCn}}{L_{s}}\left\|\sqrt{\eta^{n}} \mathbf{u}_{s}^{n+1}\right\|_{\Gamma}^{2}-\frac{\mathrm{Cn}}{\mathrm{Pe}_{s}}\left\|L^{n+1}\right\|_{\Gamma}^{2} \leq 0,
\end{aligned}
$$

where

$$
\begin{aligned}
E_{\text {tot }}^{n}= & \frac{\mathrm{We}}{2}\left(\rho^{n},\left|\mathbf{u}^{n}\right|^{2}\right)+\frac{\delta t^{2} \mathrm{We}}{2 \chi}\left\|\nabla p^{n}\right\|^{2}+\frac{\mathrm{Cn}^{2}}{2}\left\|\nabla \phi^{n}\right\|^{2}+\left(U^{n}\right)^{2}+\operatorname{Pi}\left(V^{n}\right)^{2} \\
& +\frac{1}{2 \mathrm{Ex}}\left(\psi^{n},\left|\phi^{n}\right|^{2}\right)-\frac{1}{4}\left(\psi^{n},\left|W^{n}\right|^{2}\right)+\operatorname{Cn}\left(M\left(\phi^{n}\right), 1\right)_{\Gamma}-\mathrm{Pi} B|\Omega|,
\end{aligned}
$$

here $\|\cdot\|$ denotes the $L^{2}$-norm in $\Omega$.

Proof. The difference between kinetic energies $E_{k}^{n+1}$ and $E_{k}^{n}$ is estimated $[60,61]$ as

$$
\begin{aligned}
E_{k}^{n+1} & -E_{k}^{n}=\frac{\mathrm{We}}{2}\left(\rho^{n+1},\left|\mathbf{u}^{n+1}\right|^{2}\right)-\frac{\mathrm{We}}{2}\left(\rho^{n},\left|\mathbf{u}^{n}\right|^{2}\right) \\
& =\frac{\mathrm{We}}{2}\left(\rho^{n},\left|\mathbf{u}^{n+1}\right|^{2}-\left|\mathbf{u}^{n}\right|^{2}\right)+\frac{\mathrm{We}}{2}\left(\rho^{n+1}-\rho^{n},\left|\mathbf{u}^{n+1}\right|^{2}\right) \\
& =\mathrm{We}\left(\rho^{n}\left(\mathbf{u}^{n+1}-\mathbf{u}^{n}\right), \mathbf{u}^{n+1}\right)-\frac{\mathrm{We}}{2}\left(\rho^{n},\left|\mathbf{u}^{n+1}-\mathbf{u}^{n}\right|^{2}\right)+\frac{\mathrm{We}}{2}\left(\rho^{n+1}-\rho^{n},\left|\mathbf{u}^{n+1}\right|^{2}\right) .
\end{aligned}
$$

According to (3.25), we have

$$
\begin{aligned}
& \mathrm{We} \rho^{n}\left(\mathbf{u}^{n+1}-\mathbf{u}^{n}\right)=\delta t \operatorname{CaCn} \nabla \cdot \eta^{n} D\left(\mathbf{u}^{n+1}\right)-\delta t \mathrm{We} \nabla\left(2 p^{n}-p^{n-1}\right) \\
& -\delta t \mathrm{We} \rho^{n}\left(\mathbf{u}^{n} \cdot \nabla\right) \mathbf{u}^{n+1}-\delta t \mathrm{We} \mathbf{J}^{n} \cdot \nabla \mathbf{u}^{n+1}-\frac{\mathrm{We}\left(\rho^{n+1}-\rho^{n}\right)}{2} \mathbf{u}^{n+1} \\
& -\frac{\delta t \mathrm{We}}{2} \nabla \cdot\left(\rho^{n} \mathbf{u}^{n}\right) \mathbf{u}^{n+1}-\frac{\delta t \mathrm{We}}{2} \nabla \cdot \mathbf{J}^{n} \mathbf{u}^{n+1}-\delta t\left(\phi^{n} \nabla w_{\phi}^{n+1}+\psi^{n} \nabla w_{\psi}^{n+1}\right) .
\end{aligned}
$$

By taking the $L^{2}$ inner product of (3.34) with $\mathbf{u}^{n+1}$, and using (3.33) and the following identities

$$
\begin{gathered}
\left(\left(\rho^{n} \mathbf{u}^{n} \cdot \nabla\right) \mathbf{u}^{n+1}+\frac{1}{2} \nabla \cdot\left(\rho^{n} \mathbf{u}^{n}\right) \mathbf{u}^{n+1}, \mathbf{u}^{n+1}\right)=0, \\
\left(\left(\mathbf{J}^{n} \cdot \nabla\right) \mathbf{u}^{n+1}+\frac{1}{2}\left(\nabla \cdot \mathbf{J}^{n}\right) \mathbf{u}^{n+1}, \mathbf{u}^{n+1}\right)=0 .
\end{gathered}
$$

we can derive that

$$
\begin{aligned}
E_{k}^{n+1} & -E_{k}^{n}=-\frac{\delta t \mathrm{CaCn}}{2}\left\|\sqrt{\eta^{n}} D\left(\mathbf{u}^{n+1}\right)\right\|^{2}-\delta t \mathrm{We}\left(p^{n+1}-2 p^{n}+p^{n-1}, \nabla \cdot \mathbf{u}^{n+1}\right) \\
& +\delta t \mathrm{We}\left(p^{n+1}, \nabla \cdot \mathbf{u}^{n+1}\right)-\frac{\mathrm{We}}{2}\left(\rho^{n},\left|\mathbf{u}^{n+1}-\mathbf{u}^{n}\right|^{2}\right)-\delta t\left(\phi^{n} \nabla w_{\phi}^{n+1}+\psi^{n} \nabla w_{\psi}^{n+1}, \mathbf{u}^{n+1}\right) \\
& +\delta t \operatorname{CaCn}\left(\eta^{n} \partial_{n} \mathbf{u}^{n+1}, \mathbf{u}^{n+1}\right)_{\Gamma},
\end{aligned}
$$

where the following integration by parts is used in the derivation of (3.35) 


$$
\delta t \operatorname{CaCn}\left(\nabla \cdot \eta^{n} D\left(\mathbf{u}^{n+1}\right), \mathbf{u}^{n+1}\right)=-\frac{\delta t \operatorname{CaCn}}{2}\left\|\sqrt{\eta^{n}} D\left(\mathbf{u}^{n+1}\right)\right\|^{2}+\delta t \operatorname{CaCn}\left(\eta^{n} \partial_{n} \mathbf{u}^{n+1}, \mathbf{u}^{n+1}\right)_{\Gamma} .
$$

For the boundary term in (3.35), using (3.28), we have

$$
\begin{gathered}
\delta t \operatorname{CaCn}\left(\eta^{n} \partial_{n} \mathbf{u}^{n+1}, \mathbf{u}^{n+1}\right)_{\Gamma}=\left(-\frac{\delta t \operatorname{CaCn} \eta^{n}}{L_{s}} \mathbf{u}_{s}^{n+1}+\delta t \operatorname{Cn} L^{n+1} \nabla_{\tau} \phi^{n}, \mathbf{u}_{\tau}^{n+1}\right)_{\Gamma} \\
=-\frac{\delta t \operatorname{CaCn}}{L_{s}}\left\|\sqrt{\eta^{n}} \mathbf{u}_{s}^{n+1}\right\|_{\Gamma}^{2}+\delta t \operatorname{Cn}\left(L^{n+1} \nabla_{\tau} \phi^{n}, \mathbf{u}_{\tau}^{n+1}\right)_{\Gamma} .
\end{gathered}
$$

By taking the $L^{2}$ inner product of (3.31) with $\delta t^{2} \mathrm{We}\left(p^{n+1}-2 p^{n}+p^{n-1}\right) / \chi$ and with $-\delta t^{2} \mathrm{We} p^{n+1} / \chi$ separately, we obtain

$$
\begin{aligned}
& -\frac{\delta t^{2} \mathrm{We}}{2 \chi}\left(\left\|\nabla\left(p^{n+1}-p^{n}\right)\right\|^{2}-\left\|\nabla\left(p^{n}-p^{n-1}\right)\right\|^{2}+\left\|\nabla\left(p^{n+1}-2 p^{n}+p^{n-1}\right)\right\|^{2}\right) \\
& =\delta t \operatorname{We}\left(p^{n+1}-2 p^{n}+p^{n-1}, \nabla \cdot \mathbf{u}^{n+1}\right),
\end{aligned}
$$

and

$$
\frac{\delta t^{2} \mathrm{We}}{2 \chi}\left(\left\|\nabla p^{n+1}\right\|^{2}-\left\|\nabla p^{n}\right\|^{2}+\left\|\nabla\left(p^{n+1}-p^{n}\right)\right\|^{2}\right)=-\delta t \mathrm{We}\left(p^{n+1}, \nabla \cdot \mathbf{u}^{n+1}\right) .
$$

Combining (3.37) and (3.38), yields

$$
\begin{array}{rl}
-\delta t & \mathrm{We}\left(p^{n+1}-2 p^{n}+p^{n-1}, \nabla \cdot \mathbf{u}^{n+1}\right)+\delta t \mathrm{We}\left(p^{n+1}, \nabla \cdot \mathbf{u}^{n+1}\right) \\
= & -\frac{\delta t^{2} \mathrm{We}}{2 \chi}\left(\left\|\nabla p^{n+1}\right\|^{2}-\left\|\nabla p^{n}\right\|^{2}\right)-\frac{\delta t^{2} \mathrm{We}}{2 \chi}\left\|\nabla\left(p^{n}-p^{n-1}\right)\right\|^{2} \\
& +\frac{\delta t^{2} \mathrm{We}}{2 \chi}\left\|\nabla\left(p^{n+1}-2 p^{n}+p^{n-1}\right)\right\|^{2} .
\end{array}
$$

We then take the difference of (3.31) at step $t^{n+1}$ and step $t^{n}$, pair the resulting equation with $\frac{\delta t^{2} \mathrm{We}}{2 \chi}\left(p^{n+1}-2 p^{n}+p^{n-1}\right)$, then take integration by parts for both sides to derive

$$
\frac{\delta t^{2} \mathrm{We}}{2 \chi}\left\|\nabla\left(p^{n+1}-2 p^{n}+p^{n-1}\right)\right\|^{2} \leq \frac{\chi}{2} \mathrm{We}\left\|\mathbf{u}^{n+1}-\mathbf{u}^{n}\right\|^{2} .
$$

Considering the fact that $\chi=\min \left(1, \lambda_{\rho}\right) / 2 \leq \rho^{n} / 2$, we obtain

$$
\frac{\delta t^{2} \mathrm{We}}{2 \chi}\left\|\nabla\left(p^{n+1}-2 p^{n}+p^{n-1}\right)\right\|^{2} \leq \frac{\chi}{2} \mathrm{We}\left\|\mathbf{u}^{n+1}-\mathbf{u}^{n}\right\|^{2} \leq \frac{\mathrm{We}}{4}\left(\rho^{n},\left|\mathbf{u}^{n+1}-\mathbf{u}^{n}\right|\right)^{2} .
$$

Summing up (3.35), (3.36), (3.39) and (3.40), we can derive that

$$
\begin{aligned}
& E_{k}^{n+1}-E_{k}^{n} \leq-\frac{\delta t \mathrm{CaCn}}{2}\left\|\sqrt{\eta^{n}} D\left(\mathbf{u}^{n+1}\right)\right\|^{2}-\frac{\delta t^{2} \mathrm{We}}{2 \chi}\left(\left\|\nabla p^{n+1}\right\|^{2}-\left\|\nabla p^{n}\right\|^{2}\right) \\
& -\delta t\left(\phi^{n} \nabla w_{\phi}^{n+1}+\psi^{n} \nabla w_{\psi}^{n+1}, \mathbf{u}^{n+1}\right)-\frac{\delta t \mathrm{CaCn}}{L_{s}}\left\|\sqrt{\eta^{n}} \mathbf{u}_{s}^{n+1}\right\|_{\Gamma}^{2}+\delta t \operatorname{Cn}\left(L^{n+1} \nabla_{\tau} \phi^{n}, \mathbf{u}_{\tau}^{n+1}\right)_{\Gamma} .
\end{aligned}
$$

By taking the inner product of (3.19) with $\delta t w_{\psi}^{n+1}$, we can easily derive that

$$
\left(\psi^{n+1}-\psi^{n}, w_{\psi}^{n+1}\right)+\delta t\left(\nabla \cdot\left(\mathbf{u}^{n+1} \psi^{n}\right), w_{\psi}^{n+1}\right)=-\frac{\delta t}{\mathrm{Pe}_{\psi}}\left\|\sqrt{M_{\psi}^{n}} \nabla w_{\psi}^{n+1}\right\|^{2} .
$$

By taking the inner product of (3.20) with $-\left(\psi^{n+1}-\psi^{n}\right)$, we have 


$$
-\left(\psi^{n+1}-\psi^{n}, w_{\psi}^{n+1}\right)=-\operatorname{Pi}\left(V^{n+1} a^{n}, \psi^{n+1}-\psi^{n}\right)-\frac{1}{2 \mathrm{Ex}}\left(\left|\phi^{n}\right|^{2}, \psi^{n+1}-\psi^{n}\right)
$$

$$
+\frac{1}{4}\left(\left|W^{n}\right|^{2}, \psi^{n+1}-\psi^{n}\right)
$$

2 where $a^{n}=G^{\prime}\left(\psi^{n}\right) / \sqrt{E_{v}\left(\psi^{n}\right)}$.

Taking the inner product of (3.21) with $2 \delta t \mathrm{Pi} V^{n+1}$ to obtain

$$
\operatorname{Pi}\left[\left(V^{n+1}\right)^{2}-\left(V^{n}\right)^{2}+\left(V^{n+1}-V^{n}\right)^{2}\right]=\operatorname{Pi}\left(V^{n+1} a^{n}, \psi^{n+1}-\psi^{n}\right) .
$$

Summing up (3.42) - (3.44), we get

$$
\begin{aligned}
\operatorname{Pi} & {\left[\left(V^{n+1}\right)^{2}-\left(V^{n}\right)^{2}+\left(V^{n+1}-V^{n}\right)^{2}\right]=\delta t\left(\mathbf{u}^{n+1} \psi^{n}, \nabla w_{\psi}^{n+1}\right)-\frac{\delta t}{\mathrm{Pe}_{\psi}}\left\|\sqrt{M_{\psi}^{n}} \nabla w_{\psi}^{n+1}\right\|^{2} } \\
& -\frac{1}{2 \mathrm{Ex}}\left(\left|\phi^{n}\right|^{2}, \psi^{n+1}-\psi^{n}\right)+\frac{1}{4}\left(\left|W^{n}\right|^{2}, \psi^{n+1}-\psi^{n}\right) .
\end{aligned}
$$

By taking the inner product of (3.22) with $\delta t w_{\phi}^{n+1}$, we have

$$
\left(\phi^{n+1}-\phi^{n}, w_{\phi}^{n+1}\right)+\delta t\left(\nabla \cdot\left(\mathbf{u}^{n+1} \phi^{n}\right), w_{\phi}^{n+1}\right)=-\frac{\delta t}{\mathrm{Pe}_{\phi}}\left\|\nabla w_{\phi}^{n+1}\right\|^{2} .
$$

By taking the inner product of (3.23) with $-\left(\phi^{n+1}-\phi^{n}\right)$, we can derive that

$$
\begin{aligned}
&-\left(\phi^{n+1}-\phi^{n}, w_{\phi}^{n+1}\right)=-\left(U^{n+1} b^{n}, \phi^{n+1}-\phi^{n}\right)-\mathrm{Cn}^{2}\left(\nabla \phi^{n+1}, \nabla \phi^{n+1}-\nabla \phi^{n}\right) \\
&- \frac{1}{\mathrm{Ex}}\left(\psi^{n+1} \phi^{n+1}, \phi^{n+1}-\phi^{n}\right)+\frac{1}{2}\left(\psi^{n+1} W^{n}\left(\phi^{n+1}+\phi^{n}\right), \phi^{n+1}-\phi^{n}\right) \\
&+\mathrm{Cn}^{2}\left(\partial_{n} \phi^{n+1}, \phi^{n+1}-\phi^{n}\right)_{\Gamma} \\
&=-\left(U^{n+1} b^{n}, \phi^{n+1}-\phi^{n}\right)-\frac{\mathrm{Cn}^{2}}{2}\left(\left\|\nabla \phi^{n+1}\right\|^{2}-\left\|\nabla \phi^{n}\right\|^{2}+\left\|\nabla \phi^{n+1}-\nabla \phi^{n}\right\|^{2}\right) \\
&-\frac{1}{2 \mathrm{Ex}}\left[\left(\psi^{n+1},\left|\phi^{n+1}\right|^{2}\right)-\left(\psi^{n+1},\left|\phi^{n}\right|^{2}\right)+\left(\psi^{n+1},\left|\phi^{n+1}-\phi^{n}\right|^{2}\right)\right] \\
&+\frac{1}{4}\left[\left(\psi^{n+1},\left|W^{n+1}\right|^{2}\right)-\left(\psi^{n+1},\left|W^{n}\right|^{2}\right)-\left(\psi^{n+1},\left|W^{n+1}-W^{n}\right|^{2}\right)\right] \\
&+\mathrm{Cn}^{2}\left(\partial_{n} \phi^{n+1}, \phi^{n+1}-\phi^{n}\right)_{\Gamma},
\end{aligned}
$$

11

12

13

where $b^{n}=F^{\prime}\left(\phi^{n}\right) / \sqrt{E_{u}\left(\phi^{n}\right)}$. Note that the following integration by parts is used when we derive (3.47)

$$
\mathrm{Cn}^{2}\left(\Delta \phi^{n+1}, \phi^{n+1}-\phi^{n}\right)=-\mathrm{Cn}^{2}\left(\nabla \phi^{n+1}, \nabla \phi^{n+1}-\nabla \phi^{n}\right)+\mathrm{Cn}^{2}\left(\partial_{n} \phi^{n+1}, \phi^{n+1}-\phi^{n}\right)_{\Gamma} .
$$

For the boundary term in (3.47), using (3.30), (3.27) and Taylor-expansion

$$
M^{\prime}\left(\phi^{n}\right)\left(\phi^{n+1}-\phi^{n}\right)=M\left(\phi^{n+1}\right)-M\left(\phi^{n}\right)-\frac{M^{\prime \prime}\left(\xi^{n}\right)}{2}\left(\phi^{n+1}-\phi^{n}\right)^{2},
$$

we have

$$
\begin{aligned}
\operatorname{Cn}^{2}\left(\partial_{n} \phi^{n+1}, \phi^{n+1}-\phi^{n}\right)_{\Gamma} & \\
= & \operatorname{Cn}\left(L^{n+1}, \phi^{n+1}-\phi^{n}\right)_{\Gamma}-\operatorname{Cn}\left(M^{\prime}\left(\phi^{n}\right)+S\left(\phi^{n+1}-\phi^{n}\right), \phi^{n+1}-\phi^{n}\right)_{\Gamma} \\
= & -\delta t \operatorname{Cn}\left(L^{n+1}, \mathbf{u}_{\tau}^{n+1} \cdot \nabla_{\tau} \phi^{n}\right)_{\Gamma}-\frac{\delta t \operatorname{Cn}}{\operatorname{Pe}_{s}}\left\|L^{n+1}\right\|_{\Gamma}^{2} \\
& -\operatorname{Cn}\left(M\left(\phi^{n+1}\right)-M\left(\phi^{n}\right), 1\right)_{\Gamma}-\operatorname{Cn}\left(S-\frac{M^{\prime \prime}\left(\xi^{n}\right)}{2},\left(\phi^{n+1}-\phi^{n}\right)^{2}\right)_{\Gamma}
\end{aligned}
$$


Taking the inner product of (3.24) with $2 \delta t U^{n+1}$ to obtain

$$
\left[\left(U^{n+1}\right)^{2}-\left(U^{n}\right)^{2}+\left(U^{n+1}-U^{n}\right)^{2}\right]=\left(U^{n+1} b^{n}, \phi^{n+1}-\phi^{n}\right) .
$$

Summing up (3.45) - (3.50), and dropping off some positive terms, we have

$$
\begin{aligned}
& \frac{\mathrm{Cn}^{2}}{2}\left(\left\|\nabla \phi^{n+1}\right\|^{2}-\left\|\nabla \phi^{n}\right\|^{2}\right)+\left[\left(U^{n+1}\right)^{2}-\left(U^{n}\right)^{2}\right]+\operatorname{Pi}\left[\left(V^{n+1}\right)^{2}-\left(V^{n}\right)^{2}\right] \\
& \quad+\frac{1}{2 \mathrm{Ex}}\left[\left(\psi^{n+1},\left|\phi^{n+1}\right|^{2}\right)-\left(\psi^{n},\left|\phi^{n}\right|^{2}\right)\right]-\frac{1}{4}\left[\left(\psi^{n+1},\left|W^{n+1}\right|^{2}\right)-\left(\psi^{n},\left|W^{n}\right|^{2}\right)\right] \\
& \quad+\mathrm{Cn}\left(M\left(\phi^{n+1}\right)-M\left(\phi^{n}\right), 1\right)_{\Gamma} \\
& \leq-\frac{\delta t}{\mathrm{Pe}_{\psi}}\left\|\sqrt{M_{\psi}^{n}} \nabla w_{\psi}^{n+1}\right\|^{2}-\frac{\delta t}{\mathrm{Pe}_{\phi}}\left\|\nabla w_{\phi}^{n+1}\right\|^{2}+\delta t\left(\mathbf{u}^{n+1} \psi^{n}, \nabla w_{\psi}^{n+1}\right)+\delta t\left(\mathbf{u}^{n+1} \phi^{n}, \nabla w_{\phi}^{n+1}\right) \\
& \quad-\delta t \operatorname{Cn}\left(\tilde{L}^{n+1}, \mathbf{u}_{\tau}^{n+1} \cdot \nabla_{\tau} \phi^{n}\right)_{\Gamma}-\frac{\delta t \mathrm{Cn}}{\mathrm{Pe}_{s}}\left\|\tilde{L}^{n+1}\right\|_{\Gamma}^{2}-\operatorname{Cn}\left(S-\frac{M^{\prime \prime}\left(\xi^{n}\right)}{2},\left(\phi^{n+1}-\phi^{n}\right)^{2}\right)_{\Gamma},
\end{aligned}
$$

Finally, combining (3.41) and (3.51), we arrive at

$$
\begin{aligned}
\frac{\mathrm{We}}{2}( & \left.\rho^{n+1},\left|\mathbf{u}^{n+1}\right|^{2}\right)-\frac{\mathrm{We}}{2}\left(\rho^{n},\left|\mathbf{u}^{n}\right|^{2}\right)+\frac{\delta t^{2} \mathrm{We}}{2 \chi}\left(\left\|\nabla p^{n+1}\right\|^{2}-\left\|\nabla p^{n}\right\|^{2}\right) \\
& +\frac{\mathrm{Cn}^{2}}{2}\left(\left\|\nabla \phi^{n+1}\right\|^{2}-\left\|\nabla \phi^{n}\right\|^{2}\right)+\left[\left(U^{n+1}\right)^{2}-\left(U^{n}\right)^{2}\right]+\operatorname{Pi}\left[\left(V^{n+1}\right)^{2}-\left(V^{n}\right)^{2}\right] \\
& +\frac{1}{2 \mathrm{Ex}}\left[\left(\psi^{n+1},\left|\phi^{n+1}\right|^{2}\right)-\left(\psi^{n},\left|\phi^{n}\right|^{2}\right)\right]-\frac{1}{4}\left[\left(\psi^{n+1},\left|W^{n+1}\right|^{2}\right)-\left(\psi^{n},\left|W^{n}\right|^{2}\right)\right] \\
& +\mathrm{Cn}\left(M\left(\phi^{n+1}\right)-M\left(\phi^{n}\right), 1\right)_{\Gamma} \\
\leq & -\frac{\delta t}{\mathrm{Pe}_{\psi}}\left\|\sqrt{M_{\psi}^{n}} \nabla w_{\psi}^{n+1}\right\|^{2}-\frac{\delta t}{\mathrm{Pe}_{\phi}}\left\|\nabla w_{\phi}^{n+1}\right\|^{2}-\frac{\delta t \mathrm{CaCn}}{2}\left\|\sqrt{\eta^{n}} D\left(\mathbf{u}^{n+1}\right)\right\|^{2}-\frac{\delta t \mathrm{Cn}}{\mathrm{Pe}_{s}}\left\|L^{n+1}\right\|_{\Gamma}^{2} \\
& -\frac{\delta t \mathrm{CaCn}_{\Gamma}}{L_{s}}\left\|\sqrt{\eta} \mathbf{u}_{s}^{n+1}\right\|_{\Gamma}^{2}-\mathrm{Cn}_{\Gamma}\left(S-\frac{M^{\prime \prime}\left(\xi^{n}\right)}{2},\left(\phi^{n+1}-\phi^{n}\right)^{2} \leq 0 .\right.
\end{aligned}
$$

By the assumption $S \geq L_{1} / 2$, we get the desired result.

\section{Spatial discretization}

In this section, we will use the finite difference method on staggered grids [62] to discretize the proposed semi-implicit scheme (3.19) - (3.31). The two-dimensional (2D) computational domain is $\Omega=\left[0, L_{x}\right] \times\left[0, L_{y}\right]$, where $L_{x}$ and $L_{y}$ are positive real numbers. The computational domain is divided into uniform rectangular meshes $T_{h}$, and cell centers are located at:

$$
x_{i}=\left(i-\frac{1}{2}\right) \Delta x, \quad i=1, \cdots, M, \quad y_{j}=\left(j-\frac{1}{2}\right) \Delta y, \quad j=1, \cdots, N,
$$

where $\Delta x=L_{x} / M, \Delta y=L_{y} / N$ are mesh sizes, and $M, N$ are the number of meshes along the $x$ and $y$ directions, respectively. Ghost cells can be easily included if we set $i=0, \ldots, M+1$, and $j=0, \ldots, N+1$. We can also define grid points by

$$
x_{i+1 / 2}=i \Delta x, \quad i=0, \cdots, M, \quad y_{j+1 / 2}=j \Delta y, \quad j=0, \cdots, N .
$$

The phase-field variable $\phi$, the concentration variable $\psi$, the chemical potentials $w_{\phi}$ and $w_{\psi}$, the pressure $p$, the density $\rho$ and the viscosity $\eta$ are defined at cell centers. We approximate the phase- 
field variable $\phi\left(x_{i}, y_{j}\right)$ by $\phi_{i, j}$, and other cell-centered variables can be identified in a similar way. For a $2 \mathrm{D}$ case, the velocity field $\mathbf{u}$ has two components, namely, $x$-direction velocity $u$ and $y$ direction velocity $v$. The $x$-direction velocity at each $y$-edge center $u\left(x_{i+1 / 2}, y_{j}\right)$ is identified via $u_{i+1 / 2, j}$, and the $y$-direction velocity at each $x$-edge center $v\left(x_{i}, y_{j+1 / 2}\right)$ is identified via $v_{i, j+1 / 2}$. Now, we define some standard forward and backward difference operators [62],

$$
\Delta_{x}^{+} \phi_{i, j}^{n+1}=\frac{\phi_{i+1, j}^{n+1}-\phi_{i, j}^{n+1}}{\Delta x}, \quad \Delta_{x}^{-} \phi_{i, j}^{n+1}=\frac{\phi_{i, j}^{n+1}-\phi_{i-1, j}^{n+1}}{\Delta x}, \quad \Delta_{y}^{+} \phi_{i, j}^{n+1}=\frac{\phi_{i, j+1}^{n+1}-\phi_{i, j}^{n+1}}{\Delta y}, \quad \Delta_{y}^{-} \phi_{i, j}^{n+1}=\frac{\phi_{i, j}^{n+1}-\phi_{i, j-1}^{n+1}}{\Delta y},
$$

$$
\begin{array}{cc}
\Delta_{x}^{+} u_{i+1 / 2, j}^{n+1}=\frac{u_{i+3 / 2, j}^{n+1}-u_{i+1 / 2, j}^{n+1}}{\Delta x}, & \Delta_{x}^{-} u_{i+1 / 2, j}^{n+1}=\frac{u_{i+1 / 2, j}^{n+1}-u_{i-1 / 2, j}^{n+1}}{\Delta x}, \\
\Delta_{y}^{+} u_{i+1 / 2, j}^{n+1}=\frac{u_{i+1 / 2, j+1}^{n+1}-u_{i+1 / 2, j}^{n+1}}{\Delta y}, & \Delta_{y}^{-} u_{i+1 / 2, j}^{n+1}=\frac{u_{i+1 / 2, j}^{n+1}-u_{i+1 / 2, j-1}^{n+1}}{\Delta y} .
\end{array}
$$

Other difference operators, e.g., $\Delta_{x}^{+} v_{i, j+1 / 2}^{n+1}$ and $\Delta_{y}^{+} v_{i, j+1 / 2}^{n+1}$, can also be defined similarly. The average values of cell-centered variables, e.g., $\phi$, in $x$ and $y$ directions can be obtained by

$$
\bar{\phi}_{i+1 / 2, j}^{n}=\frac{1}{2}\left(\phi_{i, j}^{n}+\phi_{i+1, j}^{n}\right), \quad \hat{\phi}_{i, j+1 / 2}^{n}=\frac{1}{2}\left(\phi_{i, j}^{n}+\phi_{i, j+1}^{n}\right) .
$$

On grid points of $T_{h}, \rho$ and $\eta$ can be approximated by

$$
\tilde{\rho}_{i+1 / 2, j+1 / 2}^{n}=\frac{1}{4}\left(\rho_{i, j}^{n}+\rho_{i+1, j}^{n}+\rho_{i, j+1}^{n}+\rho_{i+1, j+1}^{n}\right), \quad \tilde{\eta}_{i+1 / 2, j+1 / 2}^{n}=\frac{1}{4}\left(\eta_{i, j}^{n}+\eta_{i+1, j}^{n}+\eta_{i, j+1}^{n}+\eta_{i+1, j+1}^{n}\right) .
$$

The diffusive flux $\mathbf{J}^{n}$ has two components, namely, $J_{u}^{n}$ and $J_{v}^{n}$. Same as velocity components, $J_{u}^{n}$ and $J_{v}^{n}$ locate at the $y$-edge center and $x$-edge center, respectively, and they can be approximated by

$$
J_{u, i+1 / 2, j}^{n}=\frac{\lambda_{\rho}-1}{2 \mathrm{Pe}_{\phi}} M \Delta_{x}^{+} w_{\phi, i, j}^{n}, \quad J_{v, i, j+1 / 2}^{n}=\frac{\lambda_{\rho}-1}{2 \mathrm{Pe}_{\phi}} M \Delta_{y}^{+} w_{\phi, i, j}^{n} .
$$

The convection terms are computed using the second-order MINMOD scheme [63]. The MINMOD scheme can be viewed as a combination of the central difference, first-order and secondorder upwind schemes, and it not only achieves the second-order accuracy in space, but also preserves the physical properties of convection [63-65]. Some fully discrete operators are defined to simplify the discretization, such as $\nabla_{h} \cdot\left(\mathbf{u}^{n} \psi^{n}\right)_{i, j}, \nabla_{h} \cdot\left(\mathbf{u}^{n} \phi^{n}\right)_{i, j}, \nabla_{h} \cdot\left(\rho^{n} \mathbf{u}^{n} u^{n+1}\right)_{i+1 / 2, j}$, and $\nabla_{h} \cdot\left(\rho^{n} \mathbf{u}^{n} v^{n+1}\right)_{i, j+1 / 2}$. Details of the MINMOD discretization of convection terms can refer to the Appendix C.

We also define a fully discrete Laplacian operator as

$$
\Delta_{h} \phi_{i, j}^{n+1}=\frac{1}{\Delta x}\left(\Delta_{x}^{+} \phi_{i, j}^{n+1}-\Delta_{x}^{-} \phi_{i, j}^{n+1}\right)+\frac{1}{\Delta y}\left(\Delta_{y}^{+} \phi_{i, j}^{n+1}-\Delta_{y}^{-} \phi_{i, j}^{n+1}\right),
$$

and this operator can be extended to other cell-centered variables, e.g., $\Delta_{h} w_{\phi, i, j}^{n+1}$ and $\Delta_{h}\left(p_{i, j}^{n+1}-p_{i, j}^{n}\right)$. The Laplacians with non-constant diffusivity can be approximated by

$$
\begin{aligned}
\nabla_{h} \cdot\left(M_{\psi}^{n} \nabla_{h} w_{\psi}^{n+1}\right)_{i, j}= & \left(\bar{M}_{\psi, i+1 / 2, j}^{n} \Delta_{x}^{+} w_{\psi, i, j}^{n+1}-\bar{M}_{\psi, i-1 / 2, j}^{n} \Delta_{x}^{-} w_{\psi, i, j}^{n+1}\right) / \Delta x \\
& +\left(\hat{M}_{\psi, i, j+1 / 2}^{n} \Delta_{y}^{+} w_{\psi, i, j}^{n+1}-\hat{M}_{\psi, i, j-1 / 2}^{n} \Delta_{y}^{-} w_{\psi, i, j}^{n+1}\right) / \Delta y,
\end{aligned}
$$

and

$$
\begin{aligned}
\nabla_{h} \cdot\left(\eta^{n} \nabla_{h} u^{n+1}\right)_{i+1 / 2, j} & =\left(\eta_{i+1, j}^{n} \Delta_{x}^{+} u_{i+1 / 2, j}^{n+1}-\eta_{i, j}^{n} \Delta_{x}^{-} u_{i+1 / 2, j}^{n+1}\right) / \Delta x \\
& +\left(\tilde{\eta}_{i+1 / 2, j+1 / 2}^{n} \Delta_{y}^{+} u_{i+1 / 2, j}^{n+1}-\tilde{\eta}_{i+1 / 2, j-1 / 2}^{n} \Delta_{y}^{-} u_{i+1 / 2, j}^{n+1}\right) / \Delta y
\end{aligned}
$$


1 The operator $\nabla_{h}\left(\eta^{n} \nabla_{h} v^{n+1}\right)_{i, j+1 / 2}$ can also be defined in a similar way.

The computations of $\psi^{n+1}, \phi^{n+1}, \mathbf{u}^{n+1}$ and $p^{n+1}$ are totally decoupled if we replace $\mathbf{u}^{n+1}$ by $\mathbf{u}^{n}$ in (3.19), (3.22) and (3.27). At each time step, we only need to solve several linear elliptic equations. With operators defined above, the time-marching scheme can be fully discretized in a 2D domain. For simplicity, we only consider boundary conditions at top and bottom boundaries. The solution procedure involves the following four steps.

In step 1 , we use $\psi_{i, j}^{n}, \phi_{i, j}^{n}, u_{i+1 / 2, j}^{n}$ and $v_{i, j+1 / 2}^{n}$ to update $\psi_{i, j}^{n+1}$

$$
\begin{gathered}
\frac{\psi_{i, j}^{n+1}-\psi_{i, j}^{n}}{\Delta t}+\nabla_{h} \cdot\left(\mathbf{u}^{n} \psi^{n}\right)_{i, j}-\frac{1}{\mathrm{Pe}_{\psi}} \nabla_{h} \cdot\left(M_{\psi}^{n} \nabla_{h} w_{\psi}^{n+1}\right)_{i, j}=0, \\
w_{\psi, i, j}^{n+1}=\operatorname{Pi} a_{i, j}^{n} V^{n+1}+\frac{1}{2 \mathrm{Ex}}\left(\phi_{i, j}^{n}\right)^{2}-\frac{1}{4}\left(W_{i, j}^{n}\right)^{2}, \\
V^{n+1}-V^{n}=\frac{\Delta x \Delta y}{2} \sum_{i=1, j=1}^{M, N} a_{i, j}^{n}\left(\psi_{i, j}^{n+1}-\psi_{i, j}^{n}\right),
\end{gathered}
$$

where $a_{i, j}^{n}=G^{\prime}\left(\psi_{i, j}^{n}\right) / \sqrt{\Delta x \Delta y \sum_{i=1, j=1}^{M, N}\left(G\left(\psi_{i, j}^{n}\right)+B\right)}$. Boundary conditions at top and bottom boundaries are

$$
\Delta_{y}^{-} \psi_{i, j}^{n+1}=\Delta_{y}^{-} w_{\psi, i, j}^{n+1}=0, \quad i=1, \ldots, M, j=1, N+1 .
$$

In step $2, \phi_{i, j}^{n+1}$ is solved by using $\psi_{i, j}^{n+1}, \phi_{i, j}^{n}, u_{i+1 / 2, j}^{n}$ and $v_{i, j+1 / 2}^{n}$

$$
\begin{gathered}
\frac{\phi_{i, j}^{n+1}-\phi_{i, j}^{n}}{\delta t}+\nabla_{h} \cdot\left(\mathbf{u}^{n} \phi^{n}\right)_{i, j}-\frac{1}{\mathrm{Pe}_{\phi}} \Delta_{h} w_{\phi, i, j}^{n+1}=0, \\
w_{\phi, i, j}^{n+1}=b_{i, j}^{n} U^{n+1}-\mathrm{Cn}^{2} \Delta_{h} \phi_{i, j}^{n+1}+\frac{1}{\operatorname{Ex}} \psi_{i, j}^{n+1} \phi_{i, j}^{n+1}-\frac{1}{2} \psi_{i, j}^{n+1} W_{i, j}^{n}\left(\phi_{i, j}^{n+1}+\phi_{i, j}^{n}\right), \\
U^{n+1}-U^{n}=\frac{\Delta x \Delta y}{2} \sum_{i=1, j=1}^{M, N} b_{i, j}^{n}\left(\phi_{i, j}^{n+1}-\phi_{i, j}^{n}\right),
\end{gathered}
$$

where $b_{i, j}^{n}=F^{\prime}\left(\phi_{i, j}^{n}\right) / \sqrt{\Delta x \Delta y \sum_{i=1, j=1}^{M, N} F\left(\phi_{i, j}^{n}\right)}$. Boundary conditions at top and bottom boundaries are

$$
\Delta_{y}^{-} w_{\phi, i, j}^{n+1}=0, \quad i=1, \ldots, M, j=1, N+1,
$$

$$
\frac{\phi_{i, j-1 / 2}^{n+1}-\phi_{i, j-1 / 2}^{n}}{\delta t}+\left(\overline{\mathbf{u}}^{n} \cdot \nabla_{h} \phi^{n}\right)_{i, j-1 / 2}=-\frac{1}{\mathrm{Pe}_{s}} L_{i, j-1 / 2}^{n+1}, \quad i=1, \ldots, M, j=1, N+1,
$$

where $L_{i, j-1 / 2}^{n+1}= \pm \mathrm{Cn} \Delta_{y}^{-} \phi_{i, j}^{n+1}-\frac{\sqrt{2} \pi}{6} \cos \theta_{s} \cos \left(\frac{\pi \phi_{i, j-1 / 2}^{n}}{2}\right)+S\left(\phi_{i, j-1 / 2}^{n+1}-\phi_{i, j-1 / 2}^{n}\right), \quad+: j=N+1, \quad-: j=1$, and $2 \phi_{i, j-1 / 2}^{n+1}=\phi_{i, j}^{n+1}+\phi_{i, j-1}^{n+1}, \quad i=1, \ldots, M, j=1, N+1$.

It is worth emphasizing that $V^{n+1}$ and $U^{n+1}$ do not involve any extra computational cost, since they can be calculated explicitly once we obtain $\psi_{i, j}^{n+1}$ and $\phi_{i, j}^{n+1}$.

In step 3, we update $u_{i+1 / 2, j}^{n+1}$ and $v_{i, j+1 / 2}^{n+1}$ by solving 


$$
\begin{aligned}
& \frac{\bar{\rho}_{i+1 / 2, j}^{n}}{\delta t}\left(u_{i+1 / 2, j}^{n+1}-u_{i+1 / 2, j}^{n}\right)-\frac{1}{\operatorname{Re}} \nabla_{h}\left(\eta^{n} \nabla_{h} u^{n+1}\right)_{i+1 / 2, j}-\frac{1}{\operatorname{Re} \Delta x}\left(\eta_{i+1, j}^{n} \Delta_{x}^{+} u_{i+1 / 2, j}^{n+1}-\eta_{i, j}^{n} \Delta_{x}^{-} u_{i+1 / 2, j}^{n+1}\right) \\
& -\frac{1}{\operatorname{Re} \Delta y}\left(\tilde{\eta}_{i+1 / 2, j+1 / 2}^{n} \Delta_{x}^{+} v_{i, j+1 / 2}^{n+1}-\tilde{\eta}_{i+1 / 2, j-1 / 2}^{n} \Delta_{x}^{+} v_{i, j-1 / 2}^{n+1}\right)+\Delta_{x}^{+}\left(2 p_{i, j}^{n}-p_{i, j}^{n-1}\right)+\nabla_{h} \cdot\left(\rho^{n} \mathbf{u}^{n} u^{n+1}\right)_{i+1 / 2, j} \\
& +\nabla_{h} \cdot\left(\mathbf{J}^{n} u^{n+1}\right)_{i+1 / 2, j}+\frac{\left(\bar{\phi}_{i+1 / 2, j}^{n} \Delta_{x}^{+} w_{\phi, i, j}^{n+1}+\bar{\psi}_{i+1 / 2, j}^{n} \Delta_{x}^{+} w_{\psi, i, j}^{n+1}\right)}{\mathrm{We}}+\frac{\bar{\rho}_{i+1 / 2, j}^{n+1}-\bar{\rho}_{i+1 / 2, j}^{n}}{2 \delta t} u_{i+1 / 2, j}^{n+1} \\
& \quad+\frac{1}{2}\left(\Delta_{x}^{+}\left(\rho^{n} \bar{u}^{n}\right)_{i, j}+\Delta_{y}^{+}\left(\tilde{\rho}^{n} \bar{v}^{n}\right)_{i+1 / 2, j-1 / 2}+\Delta_{x}^{+} \bar{J}_{u, i, j}^{n}+\Delta_{y}^{+} \bar{J}_{v, i+1 / 2, j-1 / 2}^{n}\right) u_{i+1 / 2, j}^{n+1}=0
\end{aligned}
$$

2 and

$$
\begin{aligned}
& \frac{\hat{\rho}_{i, j+1 / 2}^{n+1}}{\delta t}\left(v_{i, j+1 / 2}^{n+1}-v_{i, j+1 / 2}^{n}\right)-\frac{1}{\operatorname{Re}} \nabla_{h} \cdot\left(\eta^{n} \nabla_{h} v^{n+1}\right)_{i, j+1 / 2}-\frac{1}{\operatorname{Re} \Delta y}\left(\eta_{i, j+1}^{n} \Delta_{y}^{+} v_{i, j+1 / 2}^{n+1}-\eta_{i, j}^{n} \Delta_{y}^{-} v_{i, j+1 / 2}^{n+1}\right) \\
& -\frac{1}{\operatorname{Re} \Delta x}\left(\tilde{\eta}_{i+1 / 2, j+1 / 2}^{n} \Delta_{x}^{+} u_{i+1 / 2, j}^{n+1}-\tilde{\eta}_{i-1 / 2, j+1 / 2}^{n} \Delta_{x}^{-} u_{i-1 / 2, j}^{n+1}\right)+\Delta_{y}^{+}\left(2 p_{i, j}^{n}-p_{i, j}^{n-1}\right)+\nabla_{h} \cdot\left(\rho^{n} \mathbf{u}^{n} v^{n+1}\right)_{i, j+1 / 2} \\
& +\nabla_{h} \cdot\left(\mathbf{J}^{n} v^{n+1}\right)_{i, j+1 / 2}+\frac{\left(\hat{\phi}_{i, j+1 / 2}^{n} \Delta_{y}^{+} w_{\phi, i, j}^{n+1}+\hat{\psi}_{i, j+1 / 2}^{n} \Delta_{y}^{+} w_{\psi, i, j}^{n+1}\right)}{\mathrm{We}}+\frac{\hat{\rho}_{i, j+1 / 2}^{n+1}-\hat{\rho}_{i, j+1 / 2}^{n}}{2 \delta t} v_{i, j+1 / 2}^{n+1} \\
& +\frac{1}{2}\left(\Delta_{x}^{+}\left(\tilde{\rho}^{n} \hat{u}^{n}\right)_{i-1 / 2, j+1 / 2}+\Delta_{y}^{+}\left(\rho^{n} \hat{v}^{n}\right)_{i, j}+\Delta_{x}^{+} \hat{J}_{u, i-1 / 2, j+1 / 2}^{n}+\Delta_{y}^{+} \hat{J}_{v, i, j}^{n}\right) v_{i, j+1 / 2}^{n+1}=0
\end{aligned}
$$

where $\rho_{i, j}^{n+1}=\left(1-\lambda_{\rho}\right) \widehat{\phi}_{i, j}^{n+1} / 2+\left(1+\lambda_{\rho}\right) / 2, \eta_{i, j}^{n+1}=\left(1-\lambda_{\eta}\right) \widehat{\phi}_{i, j}^{n+1} / 2+\left(1+\lambda_{\eta}\right) / 2$. Boundary conditions on top and bottom boundaries are

$$
v_{i, j-1 / 2}^{n+1}=0, \quad i=1, \ldots, M, j=1, N+1,
$$

$$
\text { where } L_{i+1 / 2, j-1 / 2}^{n+1}= \pm \operatorname{Cn} \Delta_{y}^{-} \bar{\phi}_{i+1 / 2, j}^{n+1}-\frac{\sqrt{2} \pi}{6} \cos \theta_{s} \cos \left(\frac{\pi \bar{\phi}_{i+1 / 2, j-1 / 2}^{n}}{2}\right)+S\left(\bar{\phi}_{i+1 / 2, j-1 / 2}^{n+1}-\bar{\phi}_{i+1 / 2, j-1 / 2}^{n}\right) \text {. }
$$

$$
\text { In step } 4, p_{i, j}^{n+1} \text { is updated by solving }
$$

$$
-\Delta_{h}\left(p_{i, j}^{n+1}-p_{i, j}^{n}\right)=-\frac{\chi}{\Delta t}\left(\Delta_{x}^{-} u_{i+1 / 2, j}^{n+1}+\Delta_{y}^{-} v_{i, j+1 / 2}^{n+1}\right),
$$

with the boundary condition $\Delta_{y}^{-} p_{i, j}^{n+1}=0, \quad i=1, \ldots, M, j=1, N+1$.

A preconditioned biconjugate gradient stabilized (BICGSTAB) method is used to solve the above variables. For variable-coefficient equations, matrices for the corresponding constantcoefficient systems are used as preconditioners. Note that this fully discrete scheme is decoupled and linear, but it is conditionally energy stable. Thus, we use small time step-sizes in numerical tests to reduce numerical errors and maintain the energy stability. Numerical results in section 4 indicate that the chosen time step-sizes can achieve the desired purpose.

\section{Numerical results}

In this section, we conduct a series of numerical experiments to investigate the effect of surfactants on the dynamics of MCL. 


\subsection{Model validation}

As we mentioned in the introduction section, most previous studies on two-phase flows with the MCL and surfactants utilized sharp interface models with an equilibrium equation of state $[3,5$, 44], and their models only considered insoluble surfactants on the interface, which were quite different from the diffuse interface model with soluble surfactants in this study. Thus, it is difficult to find an appropriate benchmark to directly validate our model and numerical scheme. The phasefield MCL model with soluble surfactants in this study can be divided into two sub-models: the phase-field MCL model and the phase-field surfactant model (no hydrodynamics), which are relatively easy to verify.

(a)

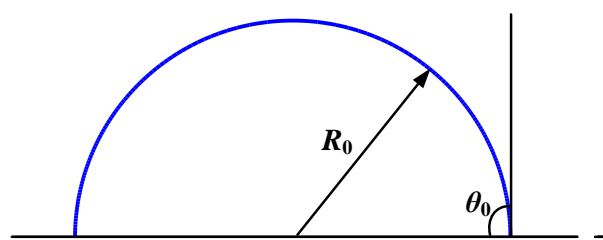

(b)

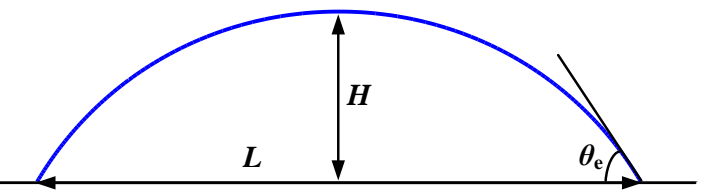

Figure 1. Illustration of (a) initial (b) equilibrium shape of the droplet. In (a), the droplet center locates at $(1,0)$. In (b), $L$ is the spreading length and $H$ is the droplet height. $\theta_{0}$ is the initial contact angle and $\theta_{e}$ is the equilibrium contact angle.

We first use a classic benchmark to validate the phase-field MCL model (no surfactants). As shown in Figure 1, the rectangular computational domain $\Omega$ is set as $[0,1] \times[0,2]$. A semicircular droplet with the radius $R_{0}=0.5$ and the initial contact angle $\theta_{0}=90^{\circ}$ is placed on the bottom surface. Periodic boundary conditions are applied in the horizontal direction, and the GNBC is imposed on the bottom surface. The droplet will spread or recoil to the equilibrium shape with the prescribed contact angle $\theta_{s}$. Note that the static contact angle $\theta_{s}$ in this study means the equilibrium contact angle $\theta_{e}$ of a clean droplet (no surfactants) on a smooth solid surface. The gravitational effect is neglected in this case. In equilibrium, the spreading length $L$ and droplet height $H$ in Figure 1(b) can be analytically obtained by the law of mass conservation [66]

$$
L=2 R_{0} \sqrt{\frac{\pi}{2\left(\theta_{e}-\sin \theta_{e} \cos \theta_{e}\right)}} \sin \theta_{e}, \quad H=R_{0} \sqrt{\frac{\pi}{2\left(\theta_{e}-\sin \theta_{e} \cos \theta_{e}\right)}}\left(1-\cos \theta_{e}\right) .
$$

We conduct several simulations in a wide range of surface wettability for both hydrophilic and hydrophobic cases $\left(\theta_{s}\right.$ varies from $45^{\circ}$ to $\left.135^{\circ}\right)$. A grid size of $300 \times 150$ and time step size $\delta t=$ $2.5 \times 10^{-4}$ are used in all simulations. Other important parameters are as follows:

$$
\mathrm{Pe}_{\phi}=10, \quad \mathrm{Re}=10, \quad \mathrm{Ca}=0.1, \quad \mathrm{Cn}=0.01, \quad \lambda_{\rho}=\lambda_{v}=1.1, \quad L_{s}=0.0038, \quad \mathrm{Pe}_{s}=0.005 .
$$

Analytical and numerical values of $L$ and $H$ are compared in Figure 2, and a good agreement is observed for all values of $\theta_{s}$. 


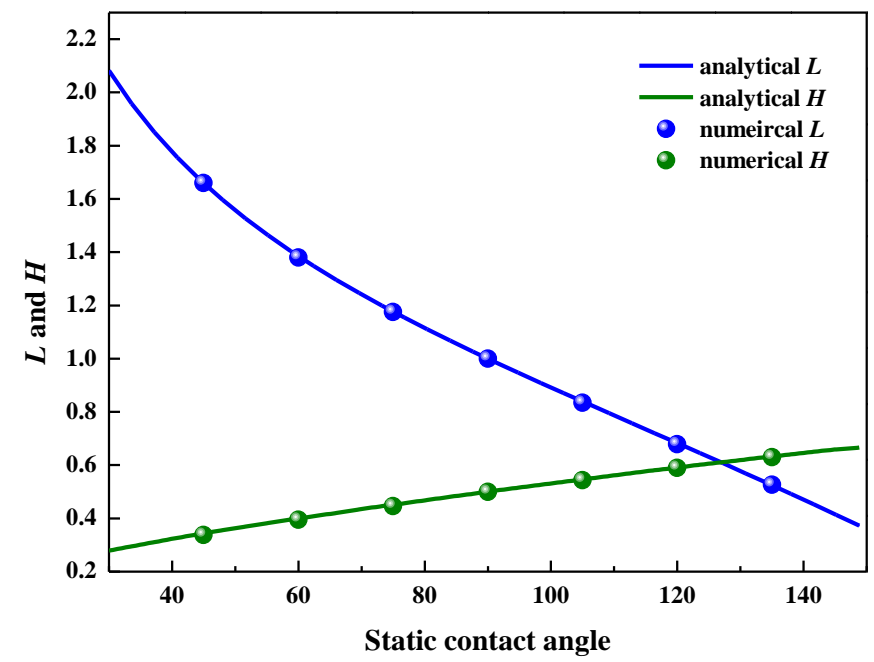

Figure 2. The comparison of analytical and numerical values of spreading length $L$ and droplet height $H$ at different static contact angles $\theta_{s} . \theta_{s}$ ranges from $45^{\circ}$ to $135^{\circ}$ with an interval $15^{\circ}$.

Next, we validate the phase-field surfactant model by comparing our results with the results obtained by the finite element-based commercial software - COMSOL [67]. There is no built-in phase-field surfactant module available in COMSOL. Thus, we use the "weak form PDE" module to realize the desired model. The computational domain $\Omega$ is set as $[0,4] \times[0,4]$. As shown in Figure 3(a), four droplets with the radius $R_{0}=0.58$ initially locate at points $(1.4,1.4),(2.6,1.4),(2.6$, 2.6) and $(1.4,2.6)$, respectively. These four droplets will merge into a circle under the effect of capillary force. A grid size of $300 \times 150$ is used in both COMSOL and in-house codes. The initial surfactant bulk concentration $\psi_{b}$ is $1 \times 10^{-2}$, and other important parameters are as follows:

$$
\mathrm{Pe}_{\phi}=10, \quad \mathrm{Pe}_{\psi}=50, \quad \mathrm{Cn}=0.03, \quad \mathrm{Ex}=1, \quad \mathrm{Pi}=0.1841, \quad \zeta=1 \times 10^{-8} .
$$
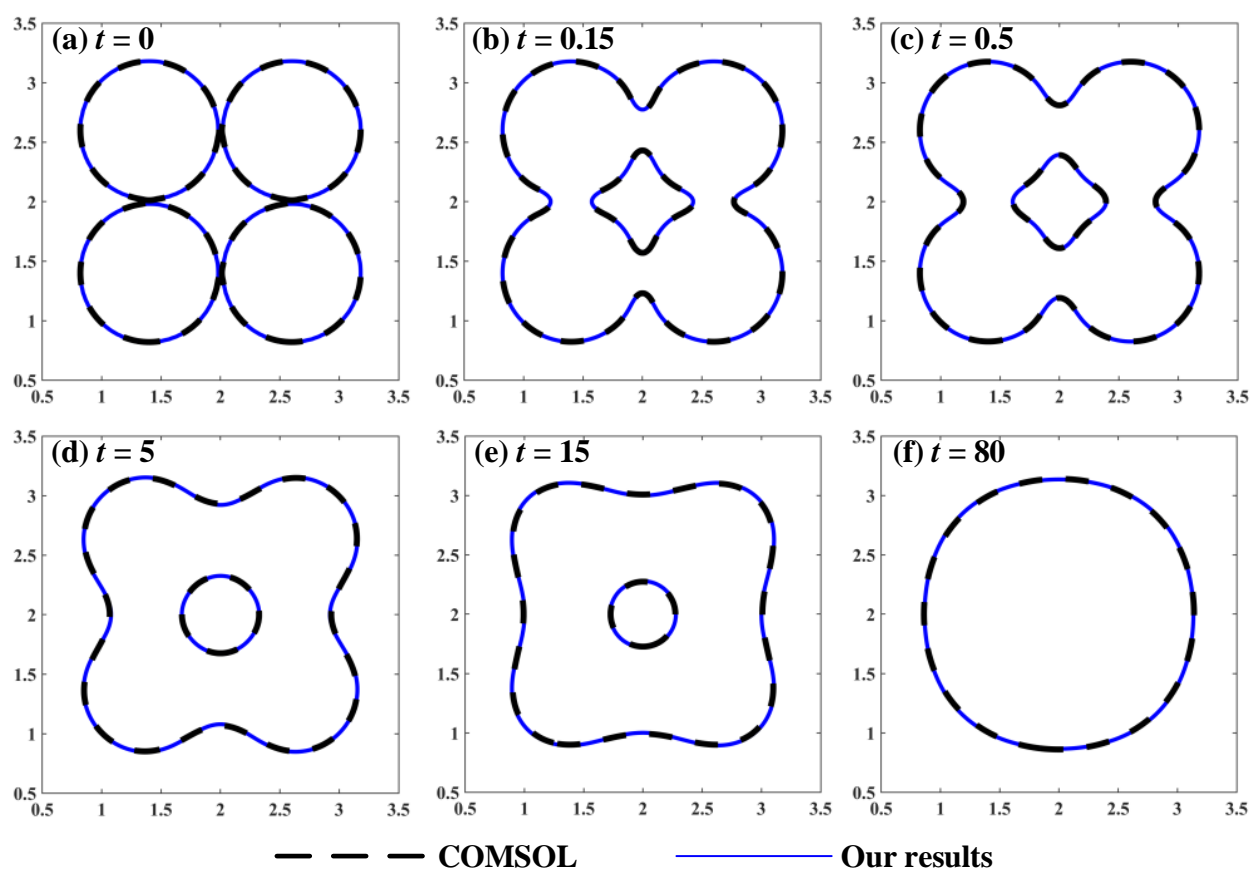

Our results

Figure 3. Droplet profiles $(\phi=0)$ at different times. The initial surfactant bulk concentration $\psi_{b}=1 \times 10^{-2}$. Black dotted 

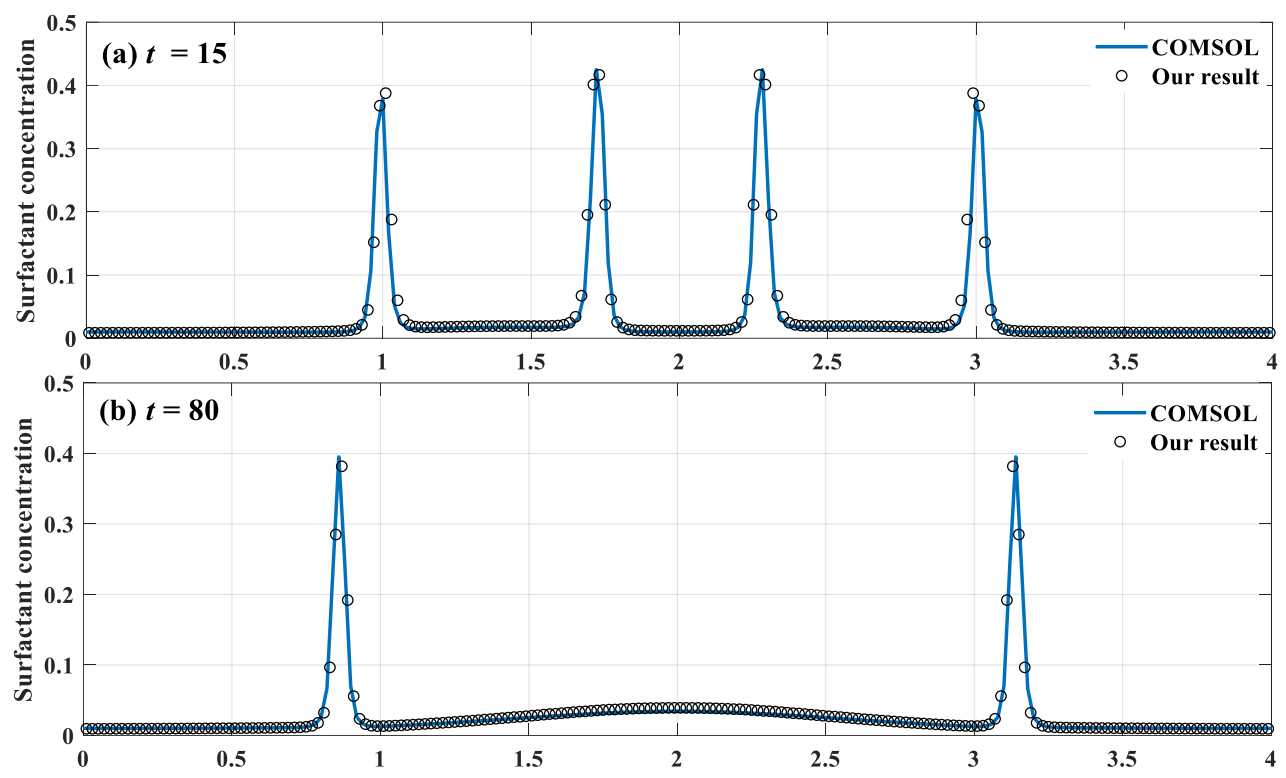

Figure 4. Surfactant concentration along a vertical line from the point $(2,0)$ to the point $(2,4)$. Blue line: results obtained by COMOSL; circle: our results.

Figure 3 gives the evolution of droplets and Figure 4 shows the surfactant concentration along the vertical line from the point $(2,0)$ to point $(2,4)$. Both of them demonstrate that our results agree well with the results obtained by COMSOL.

\subsection{Droplet spread on the solid wall}

We consider a computational domain $\Omega=[0,2] \times[0,1]$ with periodic boundary conditions on the left and right sides, as shown in Figure 5(a). The GNBCs are imposed on top and bottom walls with the static contact angle $\theta_{s}=60^{\circ}$. A semicircular droplet with a radius of 0.5 initially locates at the center of bottom wall. The initial surfactant bulk concentration $\psi_{b}$ is $1 \times 10^{-6}$. For all experiments in this section, Ex and $\psi_{c}$ are set as 1 and 0.017, respectively, with Pi determined from (2.18). Other important parameters used in simulations are as follows:

$\mathrm{Pe}_{\phi}=10, \quad \mathrm{Pe}_{\psi}=100, \quad \mathrm{Re}=10, \mathrm{Ca}=0.1, \quad \mathrm{Cn}=0.01, \quad \lambda_{\rho}=1.1, \quad \lambda_{v}=0.9, \quad L_{s}=0.0038$, $\mathrm{Pe}_{s}=0.005, \quad \zeta=1 \times 10^{-8}$.

A $300 \times 150$ grid is used in all simulations, and each simulation will run until the droplet reaches the steady state (Figure 5b). We calculate the position of the right contact point and total energy of the whole system at the time step-sizes $\delta t=4 \times 10^{-3}, 1 \times 10^{-3}, 5 \times 10^{-4}, 2.5 \times 10^{-4}$, and $6.25 \times 10^{-5}$. The solution obtained at a very small time step-size, e.g., $\delta t=6.25 \times 10^{-5}$, can serve as a reference solution. Figure 6(a) demonstrates that the position of the right contact point obtained at a large time stepsize, e.g., $\delta t=4 \times 10^{-3}$, is unacceptable because of the large numerical error. The results are reliable only when the time step-size $\delta t$ is less than $5 \times 10^{-4}$. Thus, we use the time step-size $\delta t=2.5 \times 10^{-4}$ in subsequent 2D simulations to ensure accuracy. Figure $6(\mathrm{~b})$ gives evolutions of total energy at different time step-sizes. It can be observed that the total energy is still decreasing at $\delta t=4 \times 10^{-3}$, 
1 although the numerical error is quite large. Note that these numerical results are obtained by a 2 decoupled and linear scheme in Section 4, which is conditionally energy stable. If we further 3 increase the time step-size, the total energy is no longer monotonically decreasing.
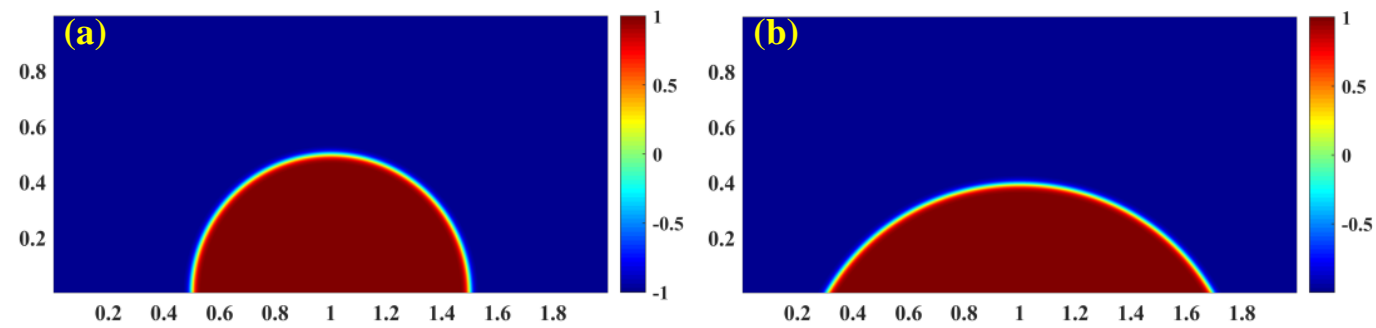

Figure 5. (a) Initial and (b) steady droplet profiles $(\phi=0)$. The initial surfactant concentration $\psi_{b}$ is $1 \times 10^{-6}$.
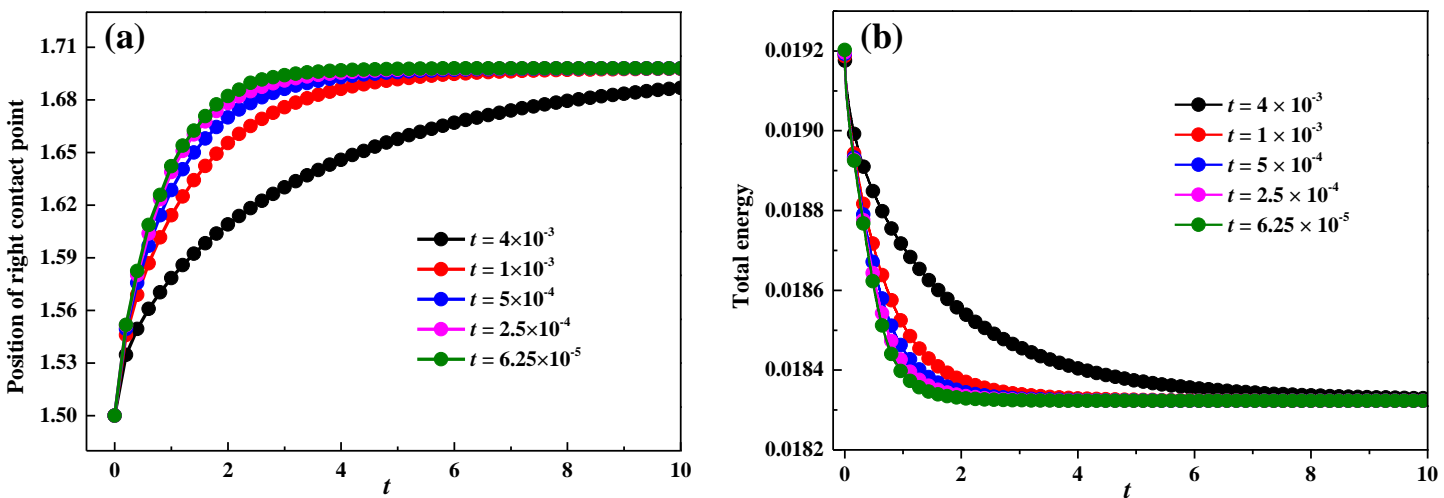

Figure 6. (a) Position of right contact point (b) evolutions of total energy $E_{t o t}$ at different time step-sizes. The initial surfactant concentration $\psi_{b}$ is $1 \times 10^{-6}$.

Now we study the effect of surfactants on the contact line dynamics. A spatial resolution $n_{x}=$ $300, n_{y}=150$ and time step-size $\delta t=2.5 \times 10^{-4}$ are used. The static contact angle $\theta_{s}$ for the bottom surface is set as $60^{\circ}$. We consider two different initial surfactant bulk concentrations $\left(\psi_{b}=1 \times 10^{-6}\right.$ and $\left.1.5 \times 10^{-2}\right)$ in simulations. Other parameters are taken as before. Figure 7 gives the evolutions of $\phi$ and $\psi$. It can be observed that surfactants are gradually migrating onto the interface, and the surfactant concentration around the interface is obviously larger than that of other area. At $t=5$, the droplet almost reaches equilibrium, and the equilibrium contact angle $\theta_{e}$ is approximately $52^{\circ}$. 

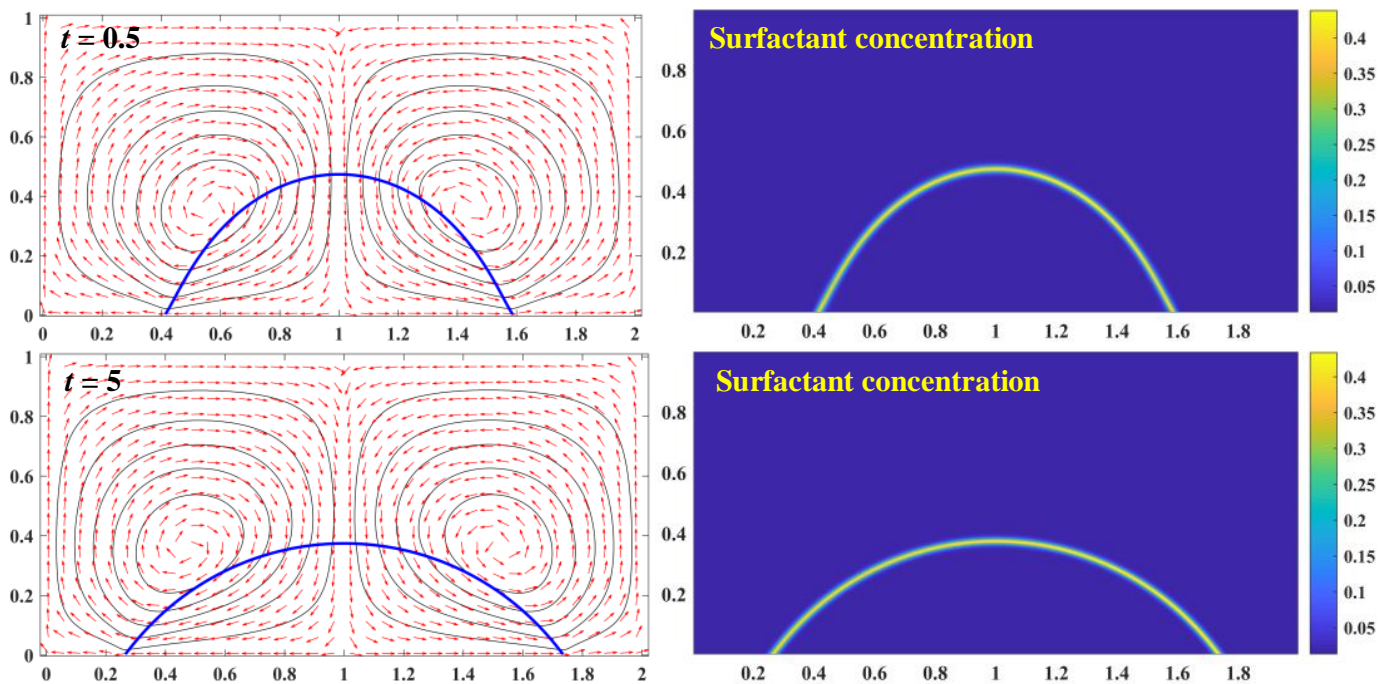

Figure 7 Streamlines, contour of $\phi=0$, quiver plot of velocity $(u, v)$ and profile of surfactant concentration $\psi$. For each subfigure, the right is the profile of $\psi \cdot\left(\psi_{b}=1.5 \times 10^{-2}\right)$.

Figure 8(a) gives the droplet profiles at different times between $t=0$ and $t=10$. The droplet constantly spreads until it reaches a steady state. The comparison of droplet profiles at different $\psi_{b}$ is presented in Figure 8(b). The equilibrium contact angles at different $\psi_{b}$ are $52^{\circ}\left(\psi_{b}=1.5 \times 10^{-2}\right)$ and $59^{\circ}\left(\psi_{b}=1 \times 10^{-6}\right)$, respectively, indicating that the increase in $\psi_{b}$ can enhance the hydrophilicity of droplet on the solid wall, and this droplet behavior can be well explained by the equation (5.2). As in [41], we can derive that

$$
\int\left(L \partial_{\tau} \phi\right) d \tau=\sigma \cos \theta_{e}+\Delta \sigma_{s}
$$

where $\int d \tau$ denotes the integration across the fluid-fluid interface along the $\tau$-direction, and $\Delta \sigma_{s}$ is a function of the local composition at the solid surface measuring the fluid-solid interfacial free energy per unit area. In equilibrium ( $L=0)$, the equation (5.2) reduces to the classic Young's equation

$$
\sigma \cos \theta_{e}+\Delta \sigma_{s}=0 .
$$

For the two cases with different $\psi_{b}, \Delta \sigma_{s}$ remains the same, since the surfactants considered in this study do not affect the fluid-solid interfacial tension through the adsorption or the like. The interfacial tension $\sigma$ at the high $\psi_{b}$ is lower than that at the low $\psi_{b}$. Thus, the droplet with a higher $\psi_{b}$ has a smaller equilibrium contact angle $\theta_{e}$ according to (5.3). Dynamics of the right contact point on the bottom wall in Figure 9 demonstrates that the droplet with the high $\psi_{b}$ spreads faster than that with the low $\psi_{b}$.
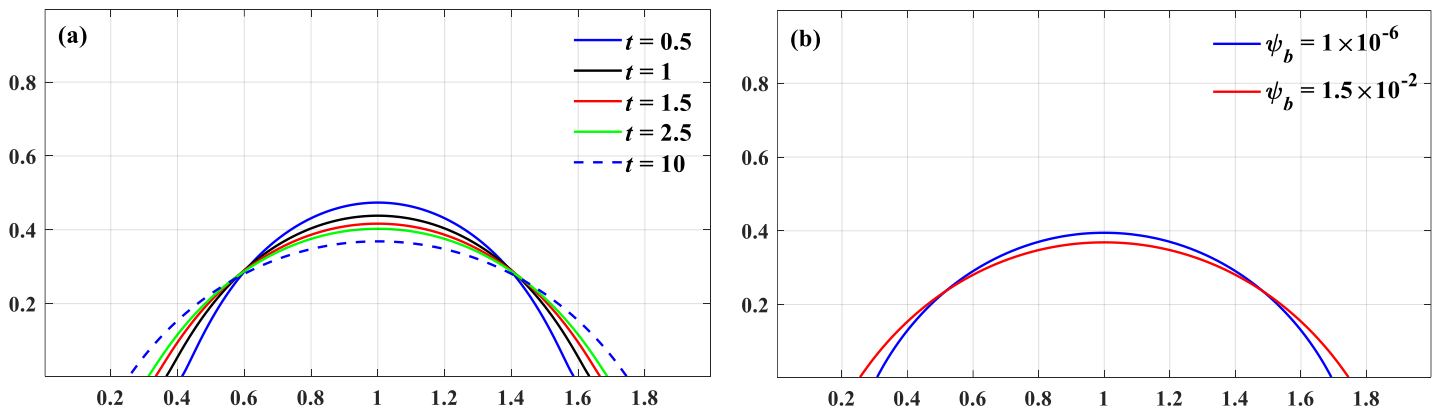

Figure 8 (a) Profiles of phase-field variable $\phi$ at different times with $\psi_{b}=1.5 \times 10^{-2}$; (b) Profiles of phase-field variable 


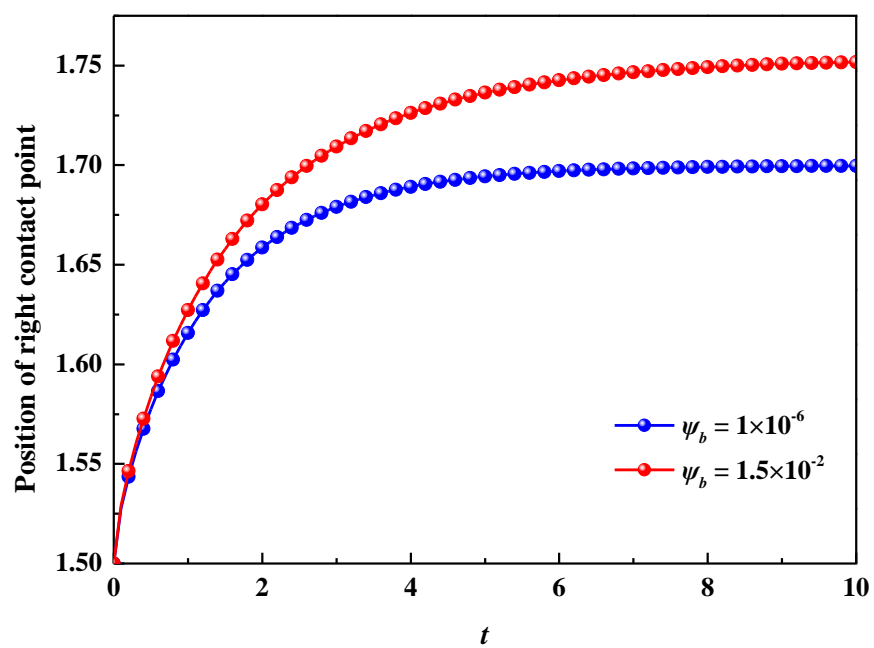

Figure 9 Position of right contact point on the solid wall at different $\psi_{b}$. (blue: $\psi_{b}=1 \times 10^{-6}$, red: $\psi_{b}=1.5 \times 10^{-2}, \theta_{s}$ $\left.=60^{\circ}\right)$

Similarly, we study the wetting behavior of a droplet on the hydrophobic surface with the static contact angle $\theta_{s}=120^{\circ}$. The unbalanced Young stress at two contact points drives the droplet to recoil until the equilibrium reaches, as shown in Figure 10(a). It can be observed in Figure 10(b) that the droplet with the high $\psi_{b}$ becomes more hydrophobic due to the reduction of interfacial tension. The trajectory of the right contact point at different $\psi_{b}$ is plotted in Figure 11. Obviously, the droplet with the high $\psi_{b}$ contracts faster.
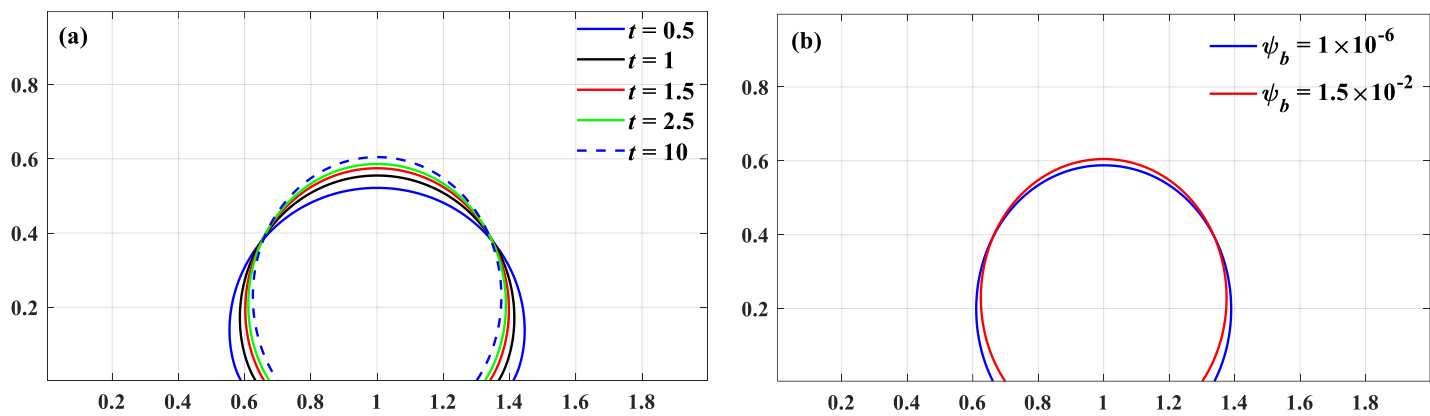

Figure 10 (a) Profiles of phase-field variable $\phi$ at different times with the initial surfactant bulk concentration $\psi_{b}=1.5 \times 10^{-2}$; (b) Profies of phase-field variable $\phi$ at $t=10$ with different $\psi_{b} .\left(\theta_{s}=120^{\circ}\right)$. 


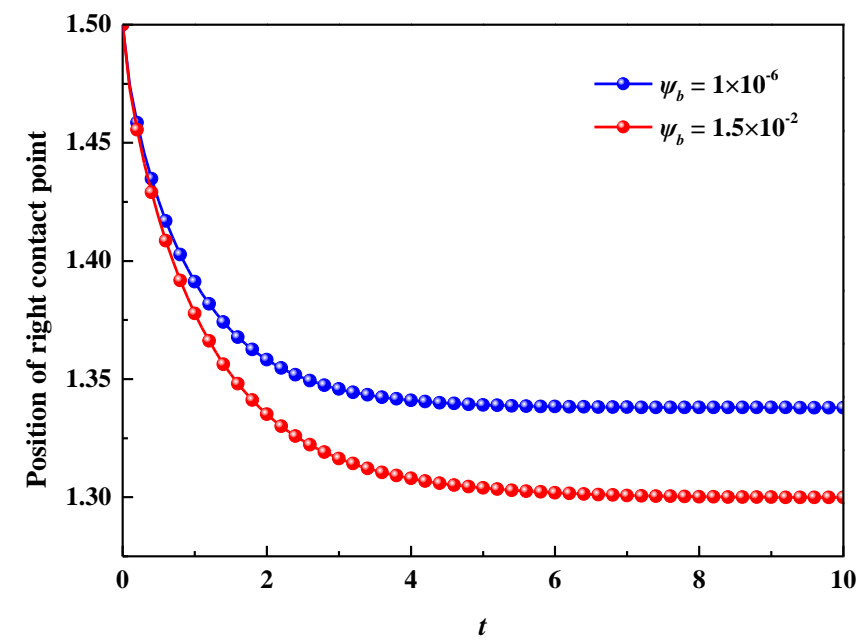

Figure 11 Position of right contact point at the different initial surfactant bulk concentrations. (blue: $\psi_{b}=1 \times 10^{-6}$, red: $\left.\psi_{b}=1.5 \times 10^{-2}, \theta_{s}=120^{\circ}\right)$

We also conduct simulations of droplet spreading and recoiling in a 3D domain $\Omega=[0,0.8]^{2} \times$ $[0,0.4]$. A $80^{2} \times 40$ grid and time step-size $\delta t=5 \times 10^{-4}$ are used in simulations. Two different $\psi_{b}$ $\left(1.5 \times 10^{-2}\right.$ and $\left.1 \times 10^{-6}\right)$ are selected as a comparison to demonstrate the effect of surfactants on the dynamics of MCL. The initial radius of the droplet is 0.25 . Péclet numbers $\mathrm{Pe}_{\phi}$ and $\mathrm{Pe}_{\psi}$ are 100 and 20, respectively. The Reynolds number Re takes 50 and Cahn number $\mathrm{Cn}$ is 0.012 . Other parameters are same as the 2D simulation. Figure 12-14 give the comparisons of droplet profiles at different $\psi_{b}$. The results of hydrophilic $\left(\theta_{s}=60^{\circ}\right)$ and hydrophobic $\left(\theta_{s}=120^{\circ}\right)$ cases are consistent with the observations in Figure 8(b) and Figure 10(b), respectively. Thus we can conclude that surfactants affect the wetting properties by altering the value of contact angles, and they make a droplet become more hydrophilic on a hydrophilic surface and more hydrophobic on a hydrophobic surface. These facts are consistent with many interesting applications in our daily life. For example, the detergent helps to remove droplets of grease from clothes by increasing contact angles.
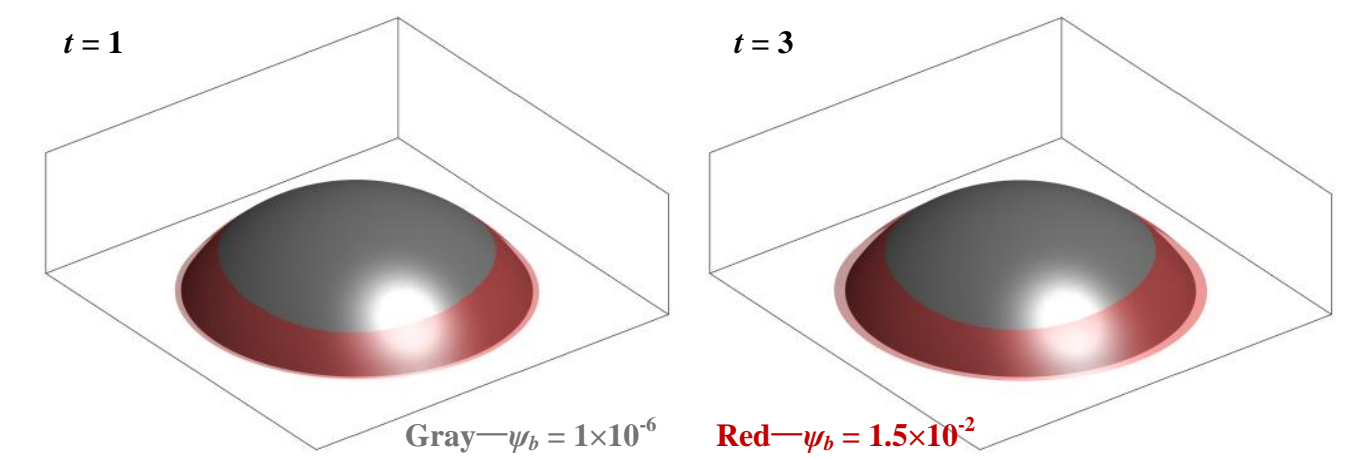

Figure 12 Comparison of droplet profiles on a hydrophilic surface $\left(\theta_{s}=60^{\circ}\right)$ at different $\psi_{b}$. (gray: $\psi_{b}=1 \times 10^{-6}$, red: $\left.\psi_{b}=1.5 \times 10^{-2}\right)$. 


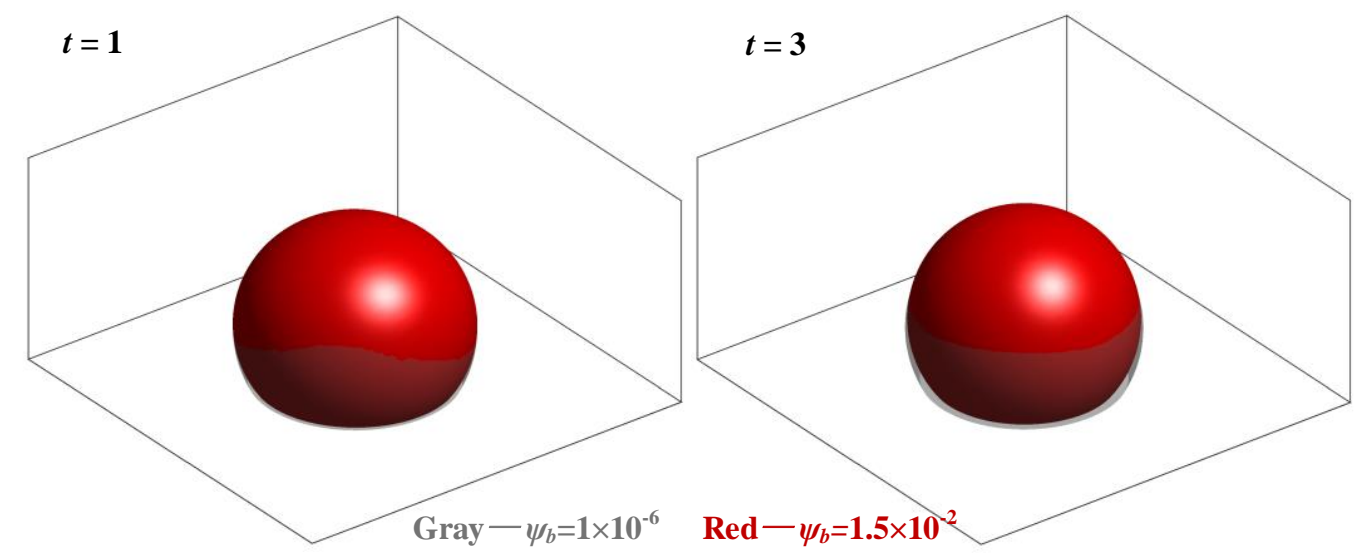

Figure 13 Comparison of droplet profiles on a hydrophobic surface $\left(\theta_{s}=120^{\circ}\right)$ at different $\psi_{b}$. (gray: $\psi_{b}=1 \times 10^{-6}$, red: $\left.\psi_{b}=1.5 \times 10^{-2}\right)$.

(a) $t=3$

$$
\operatorname{Red}-\psi_{b}=1.5 \times 10^{-2} \quad \text { Grey- } \psi_{b}=1 \times 10^{-6}
$$

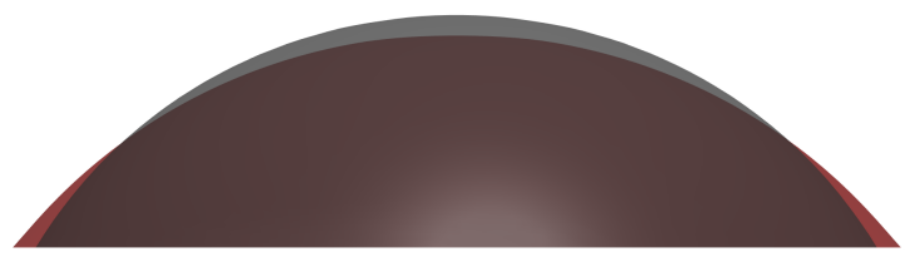

(b) $t=3$

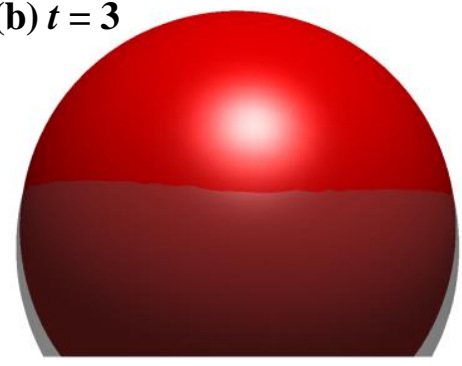

Figure 14 Comparisons of droplet profiles at different $\psi$ b. (a) hydrophilic surface $\left(\theta_{s}=60^{\circ}\right)$; (b) hydrophobic surface $\left(\theta_{s}=120^{\circ}\right)$. (gray: $\psi_{b}=1 \times 10^{-6}$, red: $\left.\psi_{b}=1.5 \times 10^{-2}\right)$.

We further extend simulations to a more complicated chemically patterned surface. The domain is $\Omega=[0,0.9]^{2} \times[0,0.4]$. A $126^{2} \times 56$ grid and time step-size $\delta t=5 \times 10^{-4}$ are adopted in our simulations. Initially, a hemispherical droplet with a radius of 0.28 is placed on the center of the patterned surface. Again, two different $\psi_{b}$ are considered in this study. Static contact angles of the droplet on gray and white zones are $\theta_{1}=60^{\circ}$ and $\theta_{2}=120^{\circ}$, respectively. Each square box has the same size $(0.1 \times 0.1)$. The evolution of the droplet is shown in Figure 15 . We can see that the droplet contracts inwards on hydrophobic zones (white) and spreads outwards on hydrophilic zones (gray) until the steady state reaches.
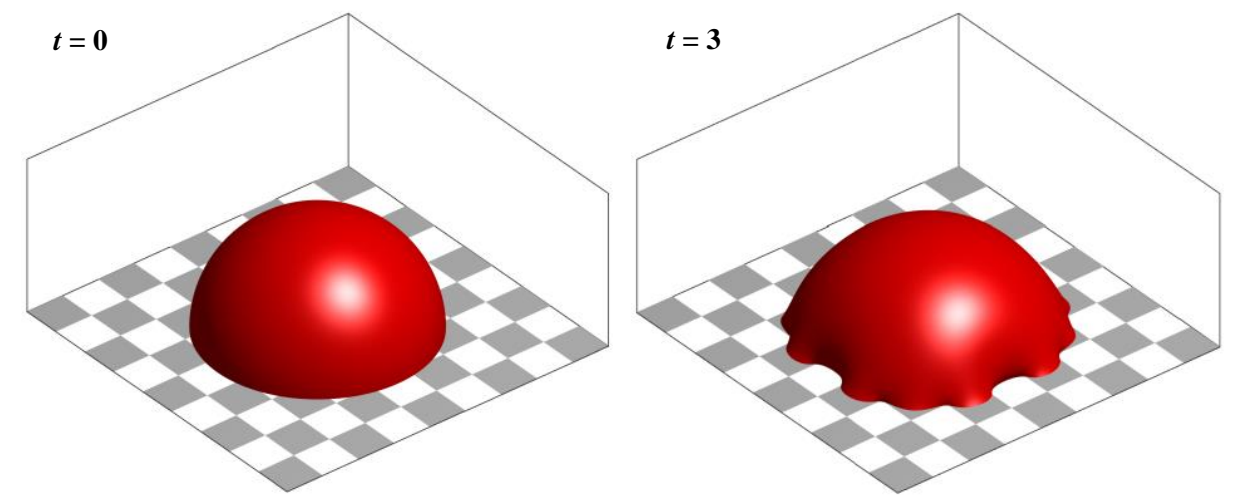

Figure 15 Evolution of a droplet on a chemically patterned surface with $\theta_{1}=60^{\circ}$ (gray zones) and $\theta_{2}=120^{\circ}$ (white 
zones $).\left(\psi_{b}=1.5 \times 10^{-2}\right)$

In Figure 16, as we expected, the large surfactant concentration leads to a smaller equilibrium contact angle on hydrophilic zones (gray), which again illustrates the effect of surfactants on the contact line dynamics.
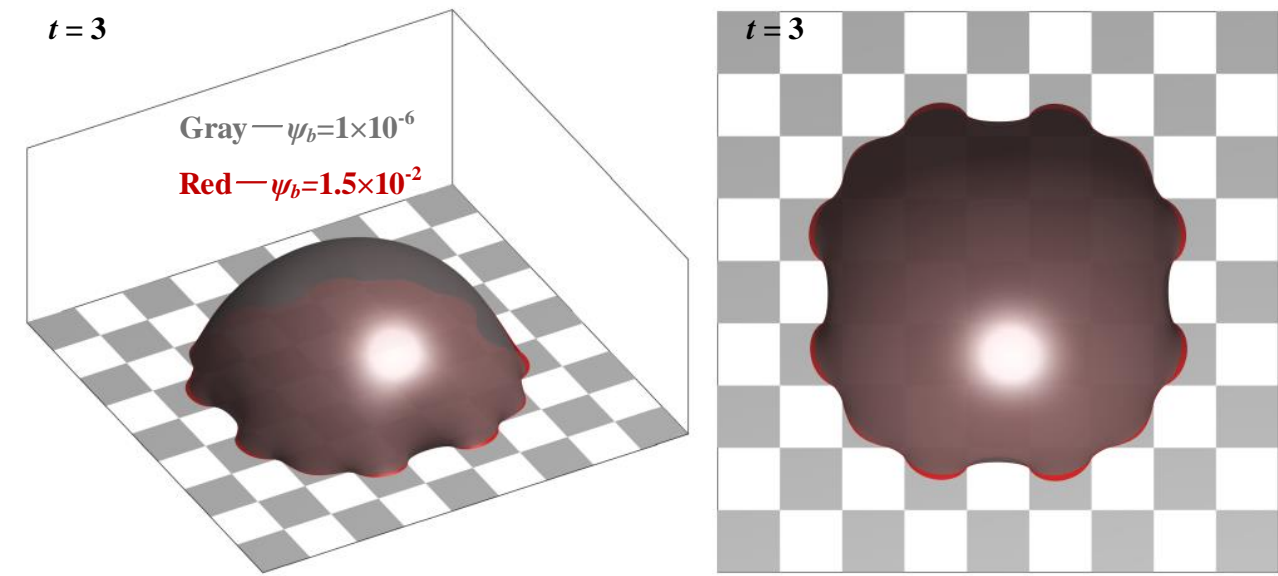

Figure 16 Comprison of droplet profiles at different $\psi_{b}$. (gray: $\psi_{b}=1 \times 10^{-6}$, red: $\psi_{b}=1.5 \times 10^{-2}$ )

It is worth noting that some conclusions obtained on a chemically smooth surface are probably not suitable for a chemically patterned surface. We can see that the difference between contact lines (contact angles) on a hydrophobic zone (the white zone, marked with a black rectangle) is not obvious in Figure 17, while our expectant result is an obvious difference between contact lines at various $\psi_{b}$. A possible reason for this phenomenon is that the adjecent gray zones significantly affect the movement of droplet on the white zone, leading to the negligible difference between contact lines.

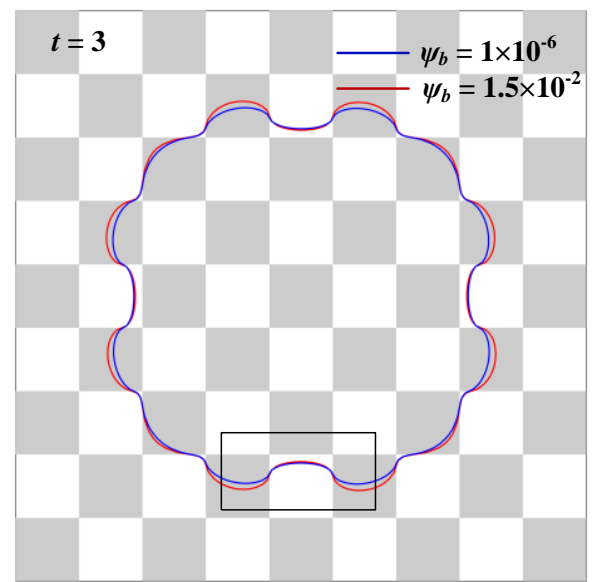

Figure 17 Position of contact lines at different $\psi_{b} .$. (blue: $\psi_{b}=1 \times 10^{-6}$, red: $\psi_{b}=1.5 \times 10^{-2}$ )

\subsection{Sliding of droplets on the solid wall}

In this section, we study the sliding of droplets driven by the horizontal body force on a solid wall. The computational domain is $\Omega=[0,4] \times[0,1]$ with periodic boundary conditions applied on the horizontal direction, as shown in Figure 18 (a). The GNBC is imposed on the bottom wall with the static contact angle $\theta_{s}=120^{\circ}$. Initially, a semicircular droplet with a radius of $r=0.5$ is located at $(1,0)$. We consider two different $\psi_{b}\left(1 \times 10^{-4}\right.$ and $\left.1 \times 10^{-2}\right)$ to investigate the effect of surfactants on 
the droplet movement. To facilitate the reader to reproduce our results, we list all parameters used in simulations:

$$
\mathrm{Pe}_{\phi}=2, \quad \mathrm{Pe}_{\psi}=100, \quad \mathrm{Re}=30, \quad \mathrm{Bo}=9, \quad \mathrm{Cn}=0.01, \quad \mathrm{Ex}=1, \quad \mathrm{Pi}=0.1841 \quad \lambda_{\rho}=0.1
$$

Figure 18 gives the evolutions of droplet profiles and surfactant concentration. As the droplet moves forward, surfactants are gradually migrating from near the wall towards the tip of droplet, as shown in Figure 18(b). The non-uniform distribution of surfactants will arise the Marangoni force [4], which will prevent the further migration of surfactants. Surfactants diffuse into the bulk phases when the tip concentration is too large. We also note that both the advancing and receding angles significantly deviate from the static contact angle $\theta_{s}=120^{\circ}$ under the effect of surfactants and the horizontal body force. The advancing angle $\theta_{A}$ in Figure $18(\mathrm{~d})$ is about $138^{\circ}$. The comparison of droplet profiles at different $\psi_{b}$ is presented in Figure 19. It can be observed that the droplet suffers the larger deformation under the high $\psi_{b}$ due to the lower interfacial tension. The advancing contact angles at different $\psi_{b}$ are $129^{\circ}$ and $138^{\circ}$ in Figure 19(d), respectively. The droplet which sticks to the solid wall becomes less "sticky" under the effect of surfactants [3], and the water can easily wash away the droplet readily.
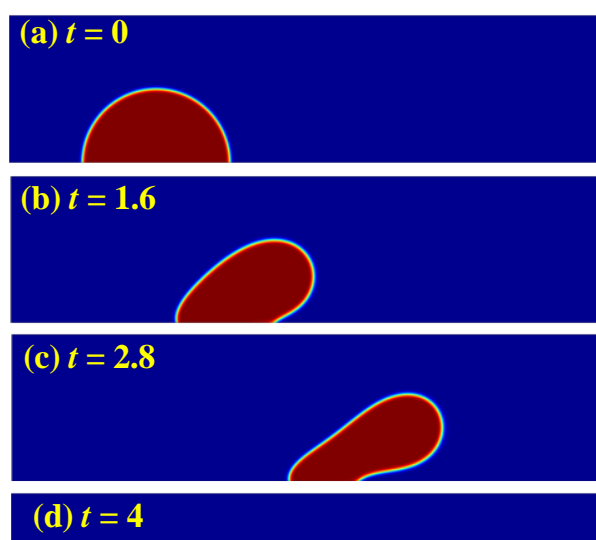

Figure 18 The movement of a droplet driven by the horizontal body force on a hydrophobic wall. For each subfigure, the left is the droplet profile and the right is the surfactant concentration. $\left(\theta_{s}=120^{\circ}\right.$ and $\left.\psi_{b}=1 \times 10^{-2}\right)$ 

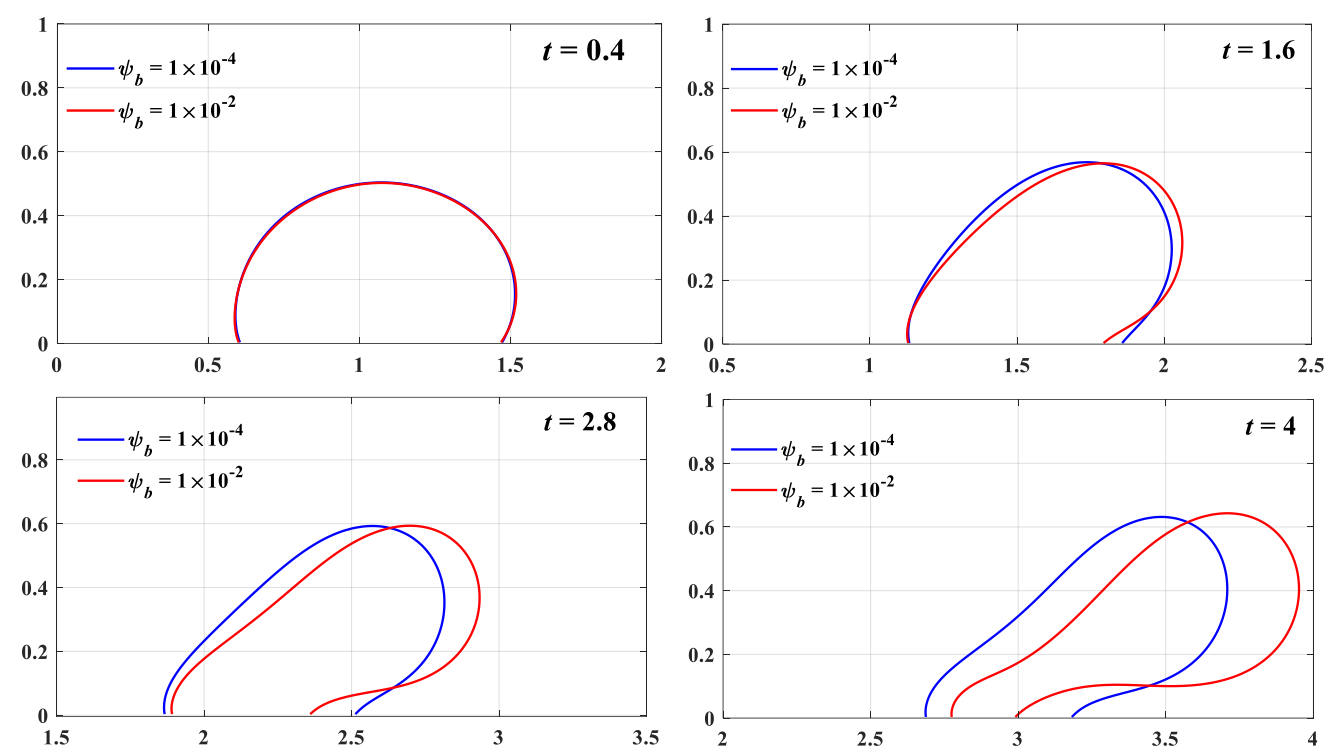

Figure 19 Comparison of droplet profiles at different initial surfactant bulk concentrations with the static contact angle $\theta_{s}=120^{\circ}$. (blue: $\psi_{b}=1 \times 10^{-4}$, red: $\psi_{b}=1 \times 10^{-2}$ )

\section{Conclusions}

In this work, a phase-field moving contact line model for a multiphase system with soluble surfactant is presented. The mathematical model consists of two Cahn-Hilliard-type equations and incompressible Navier-Stokes equations, together with the GNBC for the MCL. We construct a nonlinearly coupled energy stable scheme to solve the complex system. The scalar auxiliary variable approach is introduced to deal with nonlinear potentials and moving contact line, and a splitting method based on pressure stabilization is used to solve the Navier-Stokes equations. A subtle implicit-explicit treatment is adopted to discretize the convention and stress terms. A stabilization term is artificially added to balance the explicit nonlinear term associated with the surface energy at the fluid-solid interface. We prove the unconditional energy stability of the constructed scheme rigorously. An efficient finite difference method on staggered grids is used for the spatial discretization. Numerical results in both two and three dimensions demonstrate the accuracy and energy stability of the proposed scheme. Using our model and numerical scheme, we investigate the behavior of droplets on a solid wall. Numerical results indicate that surfactants can affect the wetting properties by altering the value of contact angles, and they make the droplet becomes more hydrophilic on a hydrophilic surface and more hydrophobic on a hydrophobic surface.

\section{Acknowledgement}

Jun Yao and Guangpu Zhu acknowledge that this work is supported by the National Science and Technology Major Project (2016ZX05011-001), the Natural Science Foundation of China (51804325, 5167428041 and 51774317) and Shandong Provincial Natural Science Foundation (ZR2019JQ21). The work of Shuyu Sun and Jisheng Kou is supported by the KAUST research fund awarded to the Computational Transport Phenomena Laboratory at KAUST through the Grant BAS/1/1351-01-01, URF/1/2993-01, and REP/1/2879-01.

\section{Appendix A}

In this section we discuss the non-dimensionalization of the phase-field MCL model with soluble surfactants. The free energy of the phase-field surfactant model in [1] is adopted in this study. 
where

$$
\left\{\begin{array}{l}
F_{1}(\phi)=-\frac{A}{2} \phi^{2}+\frac{B}{4} \phi^{4}+\frac{\kappa}{2}|\nabla \phi|^{2} \\
F_{2}(\psi)=k_{b} T[\psi \ln \psi+(1-\psi) \ln (1-\psi)] \\
F_{3}(\phi, \psi)=-\frac{\varsigma}{4} \psi\left(1-\phi^{2}\right)^{2} \\
F_{4}(\phi, \psi)=\frac{W_{c}}{2} \psi \phi^{2}
\end{array}\right.
$$

4 here parameters $A, B$ and $\kappa$ are related to the interface profile thickness $\varepsilon=\sqrt{\kappa / A}$, the two 5 homogeneous equilibrium phases $\phi_{ \pm}=\sqrt{A / B}$, and the interfacial tension $\sigma=2 \sqrt{2 \kappa A} \phi_{ \pm}^{2} / 3 \cdot k_{b}$ is the 6 Boltzmann constant, $T$ is the temperature and $W_{c}$ is a positive parameter. As in [1], we tacitly assume that $\varsigma=A$ to reduce the unnecessary free parameters.

The chemical potentials are obtained through variational derivatives of the free energy $E_{f}$

$$
\begin{gathered}
w_{\phi}=-A \phi+B \phi^{3}-\kappa \Delta \phi+W \psi \phi-\zeta \psi \phi\left(\phi^{2}-1\right), \\
w_{\psi}=k_{b} T \ln \left(\frac{\psi}{1-\psi}\right)+\frac{W}{2} \phi^{2}-\frac{\zeta}{4}\left(\phi^{2}-1\right)^{2} .
\end{gathered}
$$

Evolutions of $\phi$ and $\psi$ can be described by the conserved Cahn-Hilliard-type equations,

$$
\begin{gathered}
\phi_{t}+\nabla \cdot(\mathbf{u} \phi)=m_{\phi} \Delta w_{\phi}, \\
\psi_{t}+\nabla \cdot(\mathbf{u} \psi)=m_{\psi} \nabla \cdot M_{\psi} \nabla w_{\psi},
\end{gathered}
$$

where $m_{\phi}$ and $m_{\psi}$ are constant mobilities for $\phi$ and $\psi$, respectively. The momentum equation for the hydrodynamics takes the usual form

$$
\rho\left(\mathbf{u}_{t}+\mathbf{u} \cdot \nabla \mathbf{u}\right)+\mathbf{J} \cdot \nabla \mathbf{u}-\nabla \cdot \eta D(\mathbf{u})+\nabla p+\phi \nabla w_{\phi}+\psi \nabla w_{\psi}=0,
$$

$$
\nabla \cdot \mathbf{u}=0
$$

where

$$
\mathbf{J}=\frac{\rho_{2}-\rho_{1}}{2} m_{\phi} \nabla w_{\phi}, \quad \rho=\frac{\rho_{1}-\rho_{2}}{2} \phi+\frac{\rho_{1}+\rho_{2}}{2}, \quad \eta=\frac{\eta_{1}-\eta_{2}}{2} \phi+\frac{\eta_{1}+\eta_{2}}{2} .
$$

When the fluid-fluid interface touches the solid wall, the moving contact line problem appears. The GNBC is used to resolve the dynamics of moving contact line.

$$
\partial_{n} w_{\phi}=0, \quad \partial_{n} w_{\psi}=0, \quad \partial_{n} \psi=0, \quad \mathbf{u} \cdot \mathbf{n}=0, \quad \text { on } \Gamma,
$$

$$
\phi_{t}+\mathbf{u}_{\tau} \cdot \nabla_{\tau} \phi=-M_{\Gamma} L(\phi), \quad \text { on } \Gamma,
$$

$$
\beta \mathbf{u}_{s}+\eta \partial_{n} \mathbf{u}_{\tau}-L(\phi) \nabla_{\tau} \phi=0, \quad \text { on } \Gamma,
$$

where

$$
L(\phi)=\kappa \partial_{n} \phi-\frac{\sigma}{4} \cos \theta_{s} \cos \left(\frac{\pi \phi}{2}\right), \quad \text { on } \Gamma .
$$

here $M_{\Gamma}$ is a positive phenomenological parameter and $\beta$ is the slip coefficient. 
We define the following dimensionless parameters

$$
\mathrm{Pe}_{\phi}=\frac{L_{c} U_{c}}{m_{\phi} A}, \quad \mathrm{Pe}_{\psi}=\frac{L_{c} U_{c}}{m_{\psi} A}, \quad \operatorname{Re}=\frac{\rho_{1} L_{c} U_{c}}{\eta_{1}}, \quad \mathrm{Ca}=\frac{2 \sqrt{2} \eta_{1} U_{c}}{3 \sigma}, \quad \mathrm{Cn}=\frac{\varepsilon}{L_{c}}, \quad \lambda_{\rho}=\frac{\rho_{2}}{\rho_{1}},
$$

$$
\lambda_{\eta}=\frac{\eta_{2}}{\eta_{1}}, \quad \mathrm{Bo}=\frac{2 \sqrt{2} \rho_{1} g L_{c}^{2}}{3 \sigma}, \quad \mathrm{Ex}=\frac{\kappa}{W \varepsilon^{2}}, \quad \mathrm{Pi}=\frac{k_{b} T}{A \phi_{ \pm}^{2}}, \quad L_{s}=\frac{\eta_{1}}{\beta L_{c}}, \quad \mathrm{Pe}_{s}=\frac{\mathrm{Cn} U_{c}}{\kappa M_{\Gamma}},
$$

where $L_{c}$ is the characteristic length, $U_{c}$ is the characteristic velocity, then the characteristic time can be defined as $t_{c}=L_{c} U_{c}$. $\mathrm{Pe}_{\phi}$ and $\mathrm{Pe}_{\psi}$ are Péclet numbers. Re is the Reynolds number, $\mathrm{Ca}$ is the Capillary number, $\mathrm{Cn}$ is the Cahn number, $\mathrm{Bo}$ is the Bond number, $\mathrm{Pi}$ is a temperature-dependent constant, Ex determines the bulk solubility, and $L_{s}$ is the slip length. With these dimensionless parameters, we can easily rewrite the governing system (A.1) - (A.11) into the dimensionless form $(2.1)-(2.12)$.

Appendix B

In this section, we derive the PDE energy dissipation law for the transformed governing system (3.3) - (3.17).

Proof. With (3.6), (3.10) and (3.15), we can easily derive the following diffusive equation,

$$
\rho_{t}+\nabla \cdot(\rho \mathbf{u})+\nabla \cdot \mathbf{J}=0
$$

Using the identity (B.1), we have

$$
\begin{aligned}
& \frac{d}{d t}\left(\rho, \frac{|\mathbf{u}|^{2}}{2}\right)=\left(\rho \mathbf{u}_{t}, \mathbf{u}\right)+\left(\rho_{t}, \frac{|\mathbf{u}|^{2}}{2}\right)=\left(\rho \mathbf{u}_{t}, \mathbf{u}\right)-\left(\nabla \cdot(\rho \mathbf{u})+\nabla \cdot J, \frac{|\mathbf{u}|^{2}}{2}\right) \\
& \quad=\left(\rho \mathbf{u}_{t}+\rho \mathbf{u} \cdot \nabla \mathbf{u}+\mathbf{J} \cdot \nabla \mathbf{u}, \mathbf{u}\right),
\end{aligned}
$$

where $(\cdot, \cdot)$ is the inner product in $L^{2}(\Omega)$.

By taking the inner product of (3.9) with Weu, and using the identity (B.2), we obtain

$$
\begin{aligned}
& \frac{d}{d t}\left(\rho, \frac{\mathrm{We}|\mathbf{u}|^{2}}{2}\right)+\frac{\mathrm{CaCn}}{2}\|\sqrt{\eta} D(\mathbf{u})\|^{2}-\operatorname{CaCn}\left(\eta \partial_{n} \mathbf{u}_{\tau}, \mathbf{u}_{\tau}\right)_{\Gamma} \\
& +\left(\phi \mathbf{u}, \nabla w_{\phi}\right)+\left(\psi \mathbf{u}, \nabla w_{\psi}\right)=0
\end{aligned}
$$

where the following integration by parts is used to obtain (B.3)

$$
-\operatorname{CaCn}(\nabla \cdot \eta D(\mathbf{u}), \mathbf{u})=\frac{\mathrm{CaCn}}{2}\|\sqrt{\eta} D(\mathbf{u})\|^{2}-\operatorname{CaCn}\left(\eta \partial_{n} \mathbf{u}_{\tau}, \mathbf{u}_{\tau}\right)_{\Gamma} .
$$

For the boundary term in (B.3), using (3.13), we derive that

$$
\begin{aligned}
\operatorname{CaCn}\left(\eta \partial_{n} \mathbf{u}_{\tau}, \mathbf{u}_{\tau}\right)_{\Gamma} & =\operatorname{Cn}\left(-\frac{\operatorname{Ca} \eta}{L_{s}} \mathbf{u}_{\tau}+L(\phi) \nabla_{\tau} \phi, \mathbf{u}_{\tau}\right)_{\Gamma} \\
& =-\frac{\operatorname{CaCn}}{L_{s}}\left\|\sqrt{\eta} \mathbf{u}_{\tau}\right\|_{\Gamma}^{2}+\operatorname{Cn}\left(L(\phi) \nabla_{\tau} \phi, \mathbf{u}_{\tau}\right)_{\Gamma} .
\end{aligned}
$$

Summing up (B.3) and (B.4), we obtain 


$$
\begin{gathered}
\frac{d}{d t}\left(\rho, \frac{\mathrm{We}|\mathbf{u}|^{2}}{2}\right)+\frac{\mathrm{CaCn}}{2}\|\sqrt{\eta} D(\mathbf{u})\|^{2}+\left(\phi \mathbf{u}, \nabla w_{\phi}\right)+\left(\psi \mathbf{u}, \nabla w_{\psi}\right) \\
=-\frac{\mathrm{CaCn}}{L_{s}}\left\|\sqrt{\eta} \mathbf{u}_{\tau}\right\|_{\Gamma}^{2}+\operatorname{Cn}\left(L(\phi) \nabla_{\tau} \phi, \mathbf{u}_{\tau}\right)_{\Gamma} .
\end{gathered}
$$

By taking the inner product of (3.3) with $w_{\psi}$, we have,

$$
\left(\psi_{t}, w_{\psi}\right)-\left(\psi \mathbf{u}, \nabla w_{\psi}\right)=-\frac{1}{\mathrm{Pe}_{\psi}}\left\|\sqrt{M_{\psi}} \nabla w_{\psi}\right\|^{2} .
$$

By taking the inner product of (3.4) with $-\psi_{t}$, we can derive that

$$
-\left(w_{\psi}, \psi_{t}\right)=-\operatorname{Pi}\left(V a, \psi_{t}\right)-\frac{1}{2 \mathrm{Ex}}\left(\phi^{2}, \psi_{t}\right)+\frac{1}{4}\left(W^{2}, \psi_{t}\right),
$$

where $a=G^{\prime}(\psi) / \sqrt{E_{v}(\psi)}$.

By taking the inner product of (3.5) with $2 \mathrm{Pi} V$, we get

$$
\left(V_{t}, 2 \mathrm{Pi} V\right)=\frac{d}{d t}\left(\operatorname{Pi} V^{2}\right)=\operatorname{Pi}\left(V a, \psi_{t}\right) .
$$

Summing up (B.6) - (B.8), we obtain

$$
\frac{d}{d t}\left(\mathrm{Pi} V^{2}\right)-\left(\psi \mathbf{u}, \nabla w_{\psi}\right)=-\frac{1}{2 \mathrm{Ex}}\left(\phi^{2}, \psi_{t}\right)+\frac{1}{4}\left(W^{2}, \psi_{t}\right)-\frac{1}{\mathrm{Pe}_{\psi}}\left\|\sqrt{M_{\psi}} \nabla w_{\psi}\right\|^{2} .
$$

By taking the inner product of (3.6) with $w_{\phi}$, we get

$$
\left(\phi_{t}, w_{\phi}\right)-\left(\mathbf{u} \phi, \nabla w_{\phi}\right)=-\frac{1}{\mathrm{Pe}_{\phi}}\left\|\nabla w_{\phi}\right\|^{2} .
$$

By taking the inner product of (3.7) with $-\phi_{t}$, we can derive that

$$
-\left(w_{\phi}, \phi_{t}\right)=-\left(U b, \phi_{t}\right)-\frac{\mathrm{Cn}^{2}}{2} \frac{d}{d t}\|\nabla \phi\|^{2}+\mathrm{Cn}^{2}\left(\partial_{n} \phi, \phi_{t}\right)_{\Gamma}-\frac{1}{2 \operatorname{Ex}} \frac{d}{d t}\left(\psi, \phi^{2}\right)
$$

$$
+\frac{1}{2 \mathrm{Ex}}\left(\psi_{t}, \phi^{2}\right)+\frac{1}{4} \frac{d}{d t}\left(\psi, W^{2}\right)-\frac{1}{4}\left(\psi_{t}, W^{2}\right)
$$

where $b=F^{\prime}(\phi) / \sqrt{E_{u}(\phi)}$. The following integration by parts is used in the derivation of (B.11)

$$
\mathrm{Cn}^{2}\left(\Delta \phi, \phi_{t}\right)=-\frac{\mathrm{Cn}^{2}}{2} \frac{d}{d t}\|\nabla \phi\|^{2}+\mathrm{Cn}^{2}\left(\partial_{n} \phi, \phi_{t}\right)_{\Gamma} .
$$

For the boundary term in (B.11), using (3.14) and (3.12), we have

$$
\begin{aligned}
\operatorname{Cn}^{2}\left(\partial_{n} \phi, \phi_{t}\right)_{\Gamma} & =\operatorname{Cn}\left(L(\phi)-M^{\prime}(\phi), \phi_{t}\right)_{\Gamma} \\
= & \operatorname{Cn}\left(L(\phi),-L(\phi) / \mathrm{Pe}_{s}-\mathbf{u}_{\tau} \cdot \nabla_{\tau} \phi\right)_{\Gamma}-\operatorname{Cn} \frac{d}{d t}(M(\phi), 1)_{\Gamma} \\
= & -\frac{\mathrm{Cn}}{\mathrm{Pe}_{s}}\|L(\phi)\|_{\Gamma}^{2}-\operatorname{Cn}\left(L(\phi) \nabla_{\tau} \phi, \mathbf{u}_{\tau}\right)_{\Gamma}-\operatorname{Cn} \frac{d}{d t}(M(\phi), 1)_{\Gamma} .
\end{aligned}
$$

By taking the inner product of (3.8) with $2 U$, we have

$$
2\left(U_{t}, U\right)=\frac{d}{d t}\left(U^{2}\right)=\left(U b, \phi_{t}\right) .
$$

Combining (B.9) - (B.13), we obtain 


$$
\begin{aligned}
\frac{\mathrm{Cn}^{2}}{2} & \frac{d}{d t}\|\nabla \phi\|^{2}+\frac{1}{2 \mathrm{Ex}} \frac{d}{d t}\left(\psi, \phi^{2}\right)-\frac{1}{4} \frac{d}{d t}\left(\psi, W^{2}\right)+\operatorname{Cn} \frac{d}{d t}(M(\phi), 1)_{\Gamma}+\frac{d}{d t}\left(U^{2}+\operatorname{Pi} V^{2}\right) \\
= & \left(\psi \mathbf{u}, \nabla w_{\psi}\right)+\left(\mathbf{u} \phi, \nabla w_{\phi}\right)-\frac{1}{\mathrm{Pe}_{\psi}}\left\|\sqrt{M_{\psi}} \nabla w_{\psi}\right\|^{2}-\frac{1}{\mathrm{Pe}_{\phi}}\left\|\nabla w_{\phi}\right\|^{2} \\
& -\frac{\mathrm{Cn}}{\mathrm{Pe}_{s}}\|L(\phi)\|_{\Gamma}^{2}-\operatorname{Cn}\left(L(\phi) \nabla_{\tau} \phi, \mathbf{u}_{\tau}\right)_{\Gamma} .
\end{aligned}
$$

Summing up (B.5) and (B.14), we get the desired result (3.16)

\section{Appendix C}

The advection terms in the Cahn-Hilliard and Navier-Stokes equations are discretized by the MINMOD scheme. The deferred correction approach is used to implement the MINMOD scheme. The deferred correction procedure is a compacting technique that enables the use of high-order schemes in codes initially written for low order schemes without violating any of the stability rules $[63,68]$. We now use the advection term $\nabla \cdot\left(\rho^{n} \mathbf{u}^{n} u^{n+1}\right)_{i+1 / 2, j}$ as an example to demonstrate the discretization.
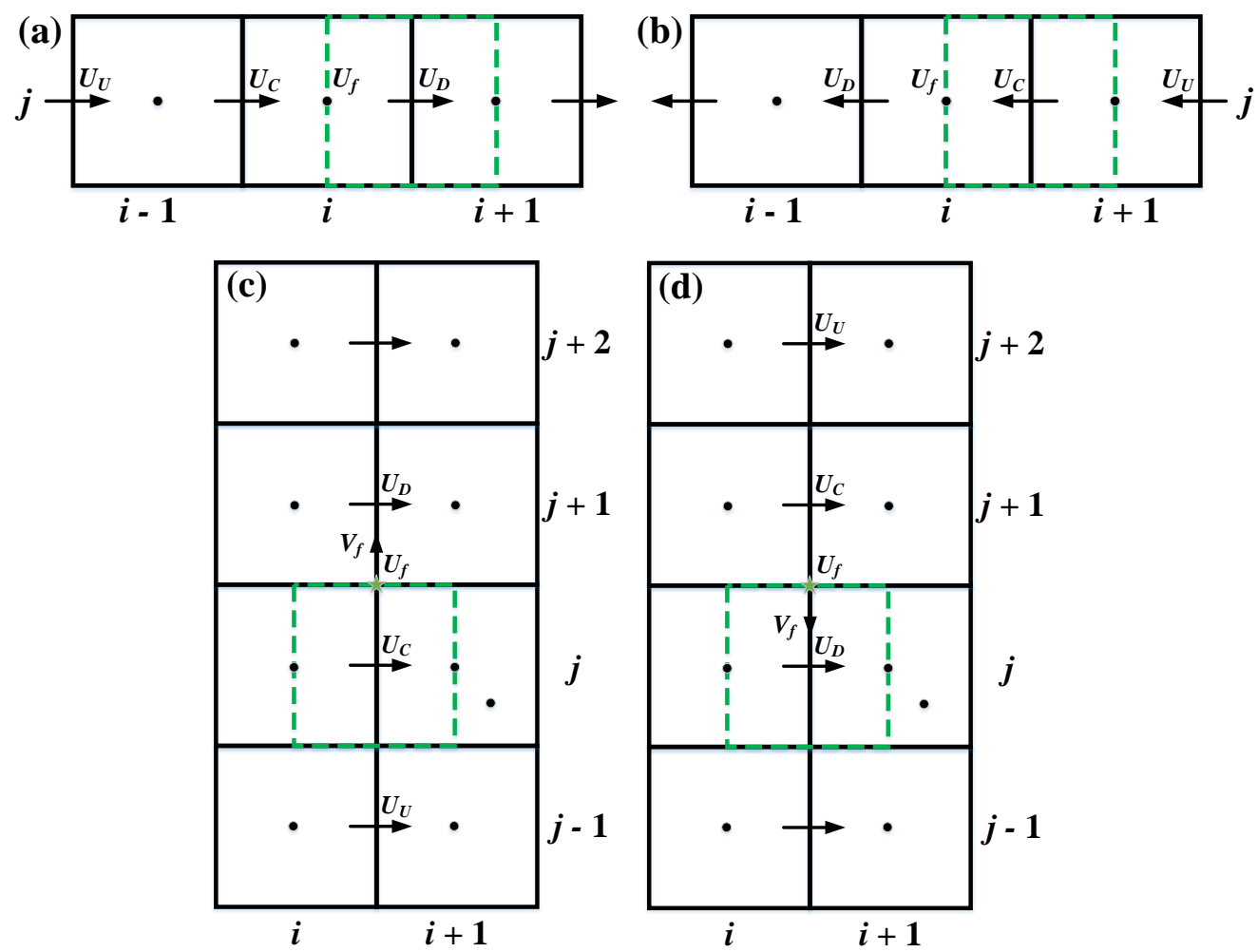

Figure S1. The shifted control volume (--, green) over which an approximation to $\nabla \cdot\left(\rho^{n} \mathbf{u}^{n} u^{n+1}\right)_{i+1 / 2, j}$ is computed. (a) The MINMOD stencil for $U_{f}^{n+1}\left(U_{f}^{n}>0\right)$; (b) the MINMOD stencil for $U_{f}^{n+1}\left(U_{f}^{n}<0\right)$; (c) The MINMOD stencil for $U_{f}^{n+1}\left(V_{f}^{n}>0\right)$; (b) the MINMOD stencil for $U_{f}^{n+1}\left(V_{f}^{n}<0\right)$.

The advection term $\nabla \cdot\left(\rho^{n} \mathbf{u}^{n} u^{n+1}\right)_{i+1 / 2, j}$ can be discretized as 


$$
\nabla_{h} \cdot\left(\rho^{n} \mathbf{u}^{n} u^{n+1}\right)_{i+1 / 2, j}=\Delta_{x}^{+}\left(\rho^{n} \bar{u}^{n} u^{n+1}\right)_{i, j}+\Delta_{x}^{+}\left(\tilde{\rho}^{n} \bar{v}^{n} u^{n+1}\right)_{i+1 / 2, j-1 / 2}
$$

$$
=\frac{\left(\rho^{n} \bar{u}^{n} u^{n+1}\right)_{i+1, j}-\left(\rho^{n} \bar{u}^{n} u^{n+1}\right)_{i, j}}{\Delta x}+\frac{\left(\tilde{\rho}^{n} \bar{v}^{n} u^{n+1}\right)_{i+1 / 2, j+1 / 2}-\left(\tilde{\rho}^{n} \bar{v}^{n} u^{n+1}\right)_{i+1 / 2, j-1 / 2}}{\Delta y} .
$$

where $u_{i, j}^{n+1}, u_{i+1, j}^{n+1}, u_{i+1 / 2, j-1 / 2}^{n+1}$ and $u_{i+1 / 2, j+1 / 2}^{n+1}$ are defined on the cell faces of the shifted control volume (Figure S1), and next they will be obtained by the MINMOD scheme. The shifted control volume centered about $x_{i+1 / 2, j}$ is constructed by shifting the computational grid by $\Delta x / 2$ in the $x$ - direction. To implement the MINMOD scheme efficiently and easily, the deferred correction approach is introduced, as presented in (C.2)

$$
u_{i, j}^{n+1, M I N}=u_{i, j}^{n+1, U p}+\left(u_{i, j}^{n, M I N}-u_{i, j}^{n, U p}\right),
$$

where superscripts $M I N$ and $U p$ refer to the MINMOD and upwind approximations, respectively.

The upwind approximation of $u_{i, j}$ on the face of the shifted control volume can be obtained by

$$
u_{i, j}^{n+1, U p}=\left\{\begin{array}{ll}
u_{i+1 / 2, j}^{n+1}, & U_{f}^{n}<0, \\
u_{i-1 / 2, j}^{n+1}, & U_{f}^{n}>0,
\end{array} \quad u_{i, j}^{n, U p}= \begin{cases}u_{i+1 / 2, j}^{n}, & U_{f}^{n}<0, \\
u_{i-1 / 2, j}^{n}, & U_{f}^{n}>0,\end{cases}\right.
$$

where $U_{f}^{n}=\bar{u}_{i, j}^{n}=\frac{1}{2}\left(u_{i+1 / 2, j}^{n}+u_{i-1 / 2, j}^{n}\right)$.

$u_{i, j}^{n, M I N}$ can be obtained by the following procedure. As shown in Figure S1, we first determine upwind $\left(U_{C}\right)$, downwind $\left(U_{D}\right)$ and far upwind $\left(U_{U}\right)$ node values depending the advection velocity $U_{f}^{n}$. If $U_{f}^{n} \geq 0$, then

$$
U_{D}=u_{i+1 / 2, j}^{n}, \quad U_{C}=u_{i-1 / 2, j}^{n}, \quad U_{U}=u_{i-3 / 2, j}^{n},
$$

and if $U_{f}^{n}<0$, we have

$$
U_{D}=u_{i-1 / 2, j}^{n}, \quad U_{C}=u_{i+1 / 2, j}^{n}, \quad U_{U}=u_{i+3 / 2, j}^{n} .
$$

With $U_{D}, U_{C}$ and $U_{U}$, we can define the normalized value through

$$
\dddot{U}=\frac{U-U_{U}}{U_{D}-U_{U}}
$$

and then the normalized MINMOD approximation of $u_{i, j}^{n}$ can be obtained by

$$
\dddot{U}_{f}= \begin{cases}\frac{3}{2} \dddot{U}_{C}, & 0 \leq \tilde{U}_{C} \leq \frac{1}{2}, \\ \frac{1}{2} \dddot{U}_{C}+\frac{1}{2}, & \frac{1}{2} \leq \tilde{U}_{C} \leq 1, \\ \dddot{U}_{C}, & \text { elsewhere. }\end{cases}
$$

With $\dddot{U}_{f}$, we can compute $u_{i, j}^{n, M I N}$ using (C.6). Substituting $u_{i, j}^{n+1, U p}, u_{i, j}^{n, U p}$ and $u_{i, j}^{n, M I N}$ into (C.2), we can obtain the desired $u_{i, j}^{n+1, M I N}$. A similar procedure can be followed to compute $u_{i+1, j}^{n+1, M I N}, u_{i+1 / 2, j-1 / 2}^{n+1, M I N}$ and $u_{i+1 / 2, j+1 / 2}^{n+1, M I N}$. Finally, we compute the approximation to $\nabla \cdot\left(\rho^{n} \mathbf{u}^{n} u^{n+1}\right)_{i+1 / 2, j}$ via

$$
\nabla_{h} \cdot\left(\rho^{n} \mathbf{u}^{n} u^{n+1}\right)_{i+1 / 2, j}=\frac{\left(\rho^{n} \bar{u}^{n}\right)_{i+1, j} u_{i+1, j}^{n+1, M I N}-\left(\rho^{n} \bar{u}^{n}\right)_{i, j} u_{i, j}^{n+1, M I N}}{\Delta x}
$$

$$
+\frac{\left(\tilde{\rho} \bar{v}^{n}\right)_{i+1 / 2, j+1 / 2} u_{i+1 / 2, j+1 / 2}^{n+1, M I N}-\left(\tilde{\rho} \bar{v}^{n}\right)_{i+1 / 2, j-1 / 2} u_{i+1 / 2, j-1 / 2}^{n+1, M I N}}{\Delta y} .
$$

Similarly, we can obtain $\nabla_{h} \cdot\left(\mathbf{u}^{n} \phi^{n}\right)_{i, j}, \nabla_{h} \cdot\left(\rho^{n} \mathbf{u}^{n} v^{n+1}\right)_{i, j+1 / 2}, \nabla_{h} \cdot\left(\mathbf{J}^{n} u^{n+1}\right)_{i+1 / 2, j}$ and so on. In 
particular, we define a operator $\left(\overline{\mathbf{u}}^{n} \cdot \nabla_{h} \phi^{n}\right)_{i, j-1 / 2}$ on the top and bottom surfaces as

$$
\left(\overline{\mathbf{u}}^{n} \cdot \nabla_{h} \phi^{n}\right)_{i, j-1 / 2}=u_{i, j-1 / 2}^{n} \frac{\phi_{i+1 / 2, j-1 / 2}^{n, M I N}-\phi_{i-1 / 2, j-1 / 2}^{n, M I N}}{\Delta x},
$$

where $i=1, \ldots, M$ and $j=1, N+1$. Note that $v_{i, j-1 / 2}^{n}=0$ on the top and bottom surfaces.

\section{References}

[1] S. Engblom, M. Do-Quang, G. Amberg, A.-K. Tornberg, On diffuse interface modeling and simulation of surfactants in two-phase fluid flow, Commun. Comput.Phys., 14 (2013) 879-915.

[2] H. Liu, Y. Zhang, Phase-field modeling droplet dynamics with soluble surfactants, J. Comput. Phys. , 229 (2010) 9166-9187.

[3] M.-C. Lai, Y.-H. Tseng, H. Huang, Numerical simulation of moving contact lines with surfactant by immersed boundary method, Commun. Comput.Phys., 8 (2010) 735.

[4] H. Liu, Y. Ba, L. Wu, Z. Li, G. Xi, Y. Zhang, A hybrid lattice Boltzmann and finite difference method for droplet dynamics with insoluble surfactants, J. Fluid Mech., 837 (2018) 381-412.

[5] J.-J. Xu, Y. Yang, J. Lowengrub, A level-set continuum method for two-phase flows with insoluble surfactant, J. Comput. Phys. , 231 (2012) 5897-5909.

[6] J.-J. Xu, W. Ren, A level-set method for two-phase flows with moving contact line and insoluble surfactant, J. Comput. Phys. , 263 (2014) 71-90.

[7] J.-J. Xu, Z. Li, J. Lowengrub, H. Zhao, A level-set method for interfacial flows with surfactant, J. Comput. Phys. , 212 (2006) 590-616.

[8] A. Alke, D. Bothe, 3D numerical modeling of soluble surfactant at fluidic interfaces based on the volume-of-fluid method, FDMP, 5 (2009) 345-372.

[9] A.J. James, J. Lowengrub, A surfactant-conserving volume-of-fluid method for interfacial flows with insoluble surfactant, J. Comput. Phys. , 201 (2004) 685-722.

[10] M. Muradoglu, G. Tryggvason, A front-tracking method for computation of interfacial flows with soluble surfactants, J. Comput. Phys. , 227 (2008) 2238-2262.

[11] R. Van der Sman, S. Van der Graaf, Diffuse interface model of surfactant adsorption onto flat and droplet interfaces, Rheol. Acta 46 (2006) 3-11.

[12] O. Theissen, G. Gompper, Lattice-Boltzmann study of spontaneous emulsification, The European Physical Journal B-Condensed Matter and Complex Systems, 11 (1999) 91-100.

[13] L. Zhang, Q. Kang, J. Yao, Y. Gao, Z. Sun, H. Liu, A.J. Valocchi, Pore scale simulation of liquid and gas two-phase flow based on digital core technology, Sci. China: Technol. Sci., 58 (2015) 1375-1384.

[14] I. Fonseca, M. Morini, V. Slastikov, Surfactants in foam stability: A phase-field model, Arch. Ration. Mech. Anal. , 183 (2007) 411-456.

[15] S. Gu, H. Zhang, Z. Zhang, An energy-stable finite-difference scheme for the binary fluid-surfactant system, J. Comput. Phys. , 270 (2014) 416-431.

[16] K.E. Teigen, P. Song, J. Lowengrub, A. Voigt, A diffuse-interface method for two-phase flows with soluble surfactants, J. Comput. Phys. , 230 (2011) 375-393.

[17] J. Kou, S. Sun, Thermodynamically consistent simulation of nonisothermal diffuse-interface twophase flow with Peng-Robinson equation of state, J. Comput. Phys. , 371 (2018) 581-605.

[18] H. Garcke, K.F. Lam, B. Stinner, Diffuse interface modelling of soluble surfactants in two-phase flow, Commun. Math. Sci., 12 (2014) 1475-1522.

[19] F. Alpak, A. Samardžić, F. Frank, A distributed parallel direct simulator for pore-scale two-phase 
flow on digital rock images using a finite difference implementation of the phase-field method, J. Petrol. Sci. Eng., 166 (2018) 806-824.

[20] F.O. Alpak, B. Riviere, F. Frank, A phase-field method for the direct simulation of two-phase flows in pore-scale media using a non-equilibrium wetting boundary condition, Computat. Geosci., 20 (2016) 881-908.

[21] J. Shen, X. Yang, Decoupled energy stable schemes for phase-field models of two-phase complex fluids, SIAM. J. Sci. Compyt., 36 (2014) B122-B145.

[22] J. Shen, X. Yang, Decoupled, energy stable schemes for phase-field models of two-phase incompressible flows, SIAM J. Numer. Anal. , 53 (2015) 279-296.

[23] D. Jacqmin, Calculation of two-phase Navier-Stokes flows using phase-field modeling, J. Comput. Phys. , 155 (1999) 96-127.

[24] P. Yue, J.J. Feng, C. Liu, J. Shen, A diffuse-interface method for simulating two-phase flows of complex fluids, J. Fluid Mech., 515 (2004) 293-317.

[25] H. Ding, P.D. Spelt, C. Shu, Diffuse interface model for incompressible two-phase flows with large density ratios, J. Comput. Phys., 226 (2007) 2078-2095.

[26] J. Shen, X. Yang, A phase-field model and its numerical approximation for two-phase incompressible flows with different densities and viscosities, SIAM. J. Sci. Compyt., 32 (2010) 1159-1179.

[27] C. Liu, B. Riviere, Numerical analysis of a discontinuous Galerkin method for Cahn-HilliardNavier-Stokes equations, arXiv preprint arXiv:1807.02725, (2018).

[28] G. Zhu, H. Chen, J. Yao, S. Sun, Efficient energy-stable schemes for the hydrodynamics coupled phase-field model, Appl. Math. Model., 70 (2019) 82-108.

[29] F. Frank, C. Liu, F.O. Alpak, S. Berg, B. Riviere, Direct numerical simulation of flow on pore-scale images using the phase-field method, SPE J., 23 (2018) 1,833-831,850.

[30] M. Laradji, H. Guo, M. Grant, M.J. Zuckermann, The effect of surfactants on the dynamics of phase separation, J. Phys.: Condens. Matter 4(1992) 6715.

[31] S. Komura, H. Kodama, Two-order-parameter model for an oil-water-surfactant system, Phys. Rev. E, 55 (1997) 1722.

[32] G. Pätzold, K. Dawson, Numerical simulation of phase separation in the presence of surfactants and hydrodynamics, Phys. Rev. E, 52 (1995) 6908.

[33] G.I. Tóth, B. Kvamme, Analysis of Ginzburg-Landau-type models of surfactant-assisted liquid phase separation, Phys. Rev. E, 91 (2015) 032404.

[34] Y. Li, J. Kim, A comparison study of phase-field models for an immiscible binary mixture with surfactant, Eur. Phys. J. B, 85 (2012) 340.

[35] X. Yang, L. Ju, Efficient linear schemes with unconditional energy stability for the phase field elastic bending energy model, Comput. Method. Appl. M., 315 (2017) 691-712.

[36] G. Zhu, J. Kou, S. Sun, J. Yao, A. Li, Decoupled, energy stable schemes for a phase-field surfactant model, Comput. Phys. Commun. , 233 (2018) 67-77.

[37] A. Yun, Y. Li, J. Kim, A new phase-field model for a water-oil-surfactant system, Appl. Math. Comput., 229 (2014) 422-432.

[38] S. Aland, A. Hahn, C. Kahle, R. Nürnberg, Comparative Simulations of Taylor Flow with Surfactants Based on Sharp-and Diffuse-Interface Methods, in: Transport Processes at Fluidic Interfaces, Springer, 2017, pp. 639-661.

[39] G. Zhu, J. Kou, S. Sun, J. Yao, A. Li, Numerical approximation of a phase-field surfactant model with fluid flow, J. Sci. Comput. , 80 (2019) 223-247. 
[40] M. Gao, X.-P. Wang, An efficient scheme for a phase field model for the moving contact line problem with variable density and viscosity, J. Comput. Phys., 272 (2014) 704-718.

[41] X.-P. Wang, T. Qian, P. Sheng, Moving contact line on chemically patterned surfaces, J. Fluid Mech., 605 (2008) 59-78.

[42] M. Gao, X.-P. Wang, A gradient stable scheme for a phase field model for the moving contact line problem, J. Comput. Phys. , 231 (2012) 1372-1386.

[43] G. Zhu, J. Yao, A. Li, H. Sun, L. Zhang, Pore-Scale Investigation of Carbon Dioxide-Enhanced Oil Recovery, Energy Fuels, 31 (2017) 5324-5332.

[44] Z. Zhang, S. Xu, W. Ren, Derivation of a continuum model and the energy law for moving contact lines with insoluble surfactants, Phys. Fluids, 26 (2014) 062103.

[45] W. Fan, H. Sun, J. Yao, D. Fan, Y. Yang, Homogenization approach for liquid flow within shale system considering slip effect, Journal of Cleaner Production, 235 (2019) 146-157.

[46] T. Qian, X.-P. Wang, P. Sheng, Molecular scale contact line hydrodynamics of immiscible flows, Phys. Rev. E, 68 (2003) 016306.

[47] T. Qian, X.-P. Wang, P. Sheng, Power-law slip profile of the moving contact line in two-phase immiscible flows, Phys. Rev. Lett. , 93 (2004) 094501.

[48] T. Qian, X.-P. Wang, P. Sheng, A variational approach to moving contact line hydrodynamics, J. Fluid Mech., 564 (2006) 333-360.

[49] H. Bonart, C. Kahle, J.-U. Repke, Comparison of Energy Stable Simulation of Moving Contact Line Problems using a Thermodynamically Consistent Cahn-Hilliard Navier-Stokes Model, arXiv preprint arXiv:1809.06689, (2018).

[50] J. Chen, S. Sun, X.-P. Wang, A numerical method for a model of two-phase flow in a coupled free flow and porous media system, J. Comput. Phys. , 268 (2014) 1-16.

[51] H. Yu, X. Yang, Numerical approximations for a phase-field moving contact line model with variable densities and viscosities, J. Comput. Phys. , 334 (2017) 665-686.

[52] J. Shen, X. Yang, H. Yu, Efficient energy stable numerical schemes for a phase field moving contact line model, J. Comput. Phys., 284 (2015) 617-630.

[53] X. Yang, L. Ju, Linear and unconditionally energy stable schemes for the binary fluid-surfactant phase field model, Comput. Method. Appl. M., 318 (2017) 1005-1029.

[54] J. Shen, J. Xu, J. Yang, The scalar auxiliary variable (SAV) approach for gradient flows, J. Comput. Phys. , 353 (2018) 407-416.

[55] J. Shen, J. Xu, J. Yang, A new class of efficient and robust energy stable schemes for gradient flows, SIAM Rev. , 61 (2019) 474-506.

[56] U.S. Fjordholm, S. Mishra, E. Tadmor, Well-balanced and energy stable schemes for the shallow water equations with discontinuous topography, J. Comput. Phys. , 230 (2011) 5587-5609.

[57] X. Yang, H. Yu, Efficient second order unconditionally stable schemes for a phase field moving contact line model using an invariant energy quadratization approach, SIAM. J. Sci. Compyt., 40 (2018) B889-B914.

[58] M. Copetti, C.M. Elliott, Numerical analysis of the Cahn-Hilliard equation with a logarithmic free energy, Numer. Math., 63 (1992) 39-65.

[59] J.-L. Guermond, A. Salgado, A splitting method for incompressible flows with variable density based on a pressure Poisson equation, J. Comput. Phys. , 228 (2009) 2834-2846.

[60] J. Kou, S. Sun, Thermodynamically consistent modeling and simulation of multi-component twophase flow with partial miscibility, Comput. Method. Appl. M., 331 (2018) 623-649. 
1 [61] J. Kou, S. Sun, X. Wang, Linearly Decoupled Energy-Stable Numerical Methods for 2 Multicomponent Two-Phase Compressible Flow, SIAM J. Numer. Anal. , 56 (2018) 3219-3248.

3 [62] H. Chen, S. Sun, T. Zhang, Energy Stability Analysis of Some Fully Discrete Numerical Schemes 4 for Incompressible Navier-Stokes Equations on Staggered Grids, J. Sci. Comput. , (2017) 1-30.

5 [63] F. Moukalled, L. Mangani, M. Darwish, The finite volume method in computational fluid dynamics, 6 An Advanced Introduction with OpenFOAM and Matlab, (2016) 3-8.

7 [64] P. Liu, X. Yan, J. Yao, S. Sun, Modeling and analysis of the acidizing process in carbonate rocks 8 using a two-phase thermal-hydrologic-chemical coupled model, Chem. Eng. Sci. , (2019).

9 [65] J. Li, B. Yu, Y. Wang, Y. Tang, H. Wang, Study on computational efficiency of composite schemes 10 for convection-diffusion equations using single-grid and multigrid methods, J. Therm. Sci. Technol. , 10 11 (2015) JTST0009-JTST0009.

12 [66] X. Cai, H. Marschall, M. Wörner, O. Deutschmann, A phase field method with adaptive mesh 13 refinement for numerical simulation of 3D wetting processes with OpenFOAM ${ }^{\circledR}$, in: 2nd International 14 Symposium on Multiscale Multiphase Process Engineering (MMPE), Hamburg, Germany, 2014.

15 [67] A. COMSOL, COMSOL Multiphysics User's Guide, version 4.3, (2012).

16 [68] P. Khosla, S. Rubin, A diagonally dominant second-order accurate implicit scheme, Comput. Fluids 17 2(1974) 207-209. 